\title{
Correlation of Solute Transfer Into Toluene and Ethylbenzene from Water and from the Gas Phase Based on the Abraham Model
}

\author{
Timothy W. Stephens ${ }^{\mathrm{a}}$, Matthew Loera ${ }^{\mathrm{a}}$, Amanda N. Quay ${ }^{\mathrm{a}}$, Vicky Chou ${ }^{\mathrm{a}}$, Connie Shen ${ }^{\mathrm{a}}$, \\ Anastasia Wilson $^{\mathrm{a}}$, William E. Acree, Jr. ${ }^{\mathrm{a}, *}$ and Michael H. Abraham ${ }^{\mathrm{b}}$ \\ ${ }^{a}$ Department of Chemistry, 1155 Union Circle \# 305070, University of North Texas, Denton, TX 76203-5017, USA \\ ${ }^{b}$ Department of Chemistry, University College London, 20 Gordon Street, London, WC1H OAJ, UK
}

\begin{abstract}
Experimental data have been compiled from the published literature on the partition coefficients of solutes and vapors into ethylbenzene and toluene at $298 \mathrm{~K}$. The logarithms of the water-to-ethylbenzene and water-to-toluene partition coefficients, $\log P$, and gas-to-ethylbenzene and gas-to-toluene partition coefficients, $\log K$, were correlated with the Abraham solvation parameter model. The derived mathematical expressions described the observed $\log P$ and $\log K$ data for the two aromatic hydrocarbon solvents to within average deviations of 0.13 log units or less.
\end{abstract}

Keywords: Partition coefficients, infinite dilution activity coefficients, toluene, ethylbenzene, Abraham model correlations.

\section{INTRODUCTION}

Extraction provides a convenient experimental method for removing phenolic compounds, flavonoids and anthocyanidins from medicinal plants, for pre-concentrating trace chemical pollutants from environmental samples prior to chemical analysis, and for isolating active pharmaceutical ingredients and metabolites from tissue samples. Extraction methods are based on the equilibrium solute partitioning in a biphasic system containing both a liquid and condensed phase, or in the case of liquid-liquid extraction containing two or more liquid solvents having limited mutual solubility. Molecular interactions between the dissolved solute(s) and surrounding extraction solvents determine the solute's affinity for a given phase, which in turn affects both the solute recovery factor and chemical separation efficiency. Design of an effective extraction method requires complete (or nearly complete) solute recovery and large separation efficiency. Considerable effort has been given in recent years to developing solution models to enable researchers to select the best extraction solvent to achieve a desired chemical separation.

Our efforts in this regard have been directed towards deriving Abraham model correlations for predicting the logarithms of the gas-to-organic solvent partition coefficients $(\log K)$, logarithms of the water-to-organic solvent partition coefficients $(\log P)$, and logarithms of molar solubility ratios ( $\log C_{\text {solute,organic }} d C_{\text {solute, water }}$ and $C_{\text {solute,organid }} / C_{\text {solute,gas }}$ ) defined

*Address correspondence to this author at the Department of Chemistry, 1155 Union Circle \# 305070, University of North Texas, Denton, TX 76203-5017, USA; Tel: 940-565-3515; Fax: 940-565-4318;

E-mail: acree@unt.edu as the solute's molar solubility in the given organic solvent $\left(C_{\text {solute, organic }}\right)$ divided by the solute's molar solubility in water $\left(C_{\text {solute,water }}\right)$ or solute's molar solubility in the vapor phase $\left(C_{\text {solute,gas }}\right)$. The latter quantity is calculable from the equilibrium vapor pressure of the solute above its pure condensed phase. To date we and other research groups have determined Abraham model correlations for more than 70 common organic solvents [1-8], as well as the partition coefficient data for solutes dissolved in 30 room temperature ionic liquids [9-15] and in human and cow milk [16], for solutes adsorbed onto (partitioned into) polydimethylsiloxane [17, 18], polyoxymethylene [19] and polyacrylate fibers [20], and for solutes in two 1,2-dichloroethane/polar organic solvent $[21,22]$, in ten alkane/polar organic solvent [21, 23-29], and in four diisopentyl ether/polar organic solvent $[21-23,29]$ biphasic partitioning systems. The latter biphasic organic systems are needed for compounds that are not stable in an aqueous environment, or are too insoluble in water to enable an accurate measurement of the water-to-organic solvent partition coefficient which might used in calculating solute descriptors for future log $P$ predictions.

The Abraham solvation parameter model depends on two linear free energy relationships, the first for transfer processes between two condensed phases [2, 4-8, 30, 31]:

$\log \left(P\right.$ or $\left.C_{\text {solute,organic }} / C_{\text {solute,water }}\right)=\mathrm{c}_{\mathrm{p}}+\mathrm{e}_{\mathrm{p}} \cdot \mathbf{E}+\mathrm{s}_{\mathrm{p}} \cdot \mathbf{S}+\mathrm{a}_{\mathrm{p}} \cdot \mathbf{A}+$ $\mathrm{b}_{\mathrm{p}} \cdot \mathbf{B}+\mathrm{v}_{\mathrm{p}} \cdot \mathbf{V}$

and the second process involving solute transfer from the gas phase:

$\log \left(K\right.$ or $\left.C_{\text {solute,organic }} / C_{\text {solute,air }}\right)=\mathrm{c}_{\mathrm{k}}+\mathrm{e}_{\mathrm{k}} \cdot \mathbf{E}+\mathrm{s}_{\mathrm{k}} \cdot \mathbf{S}+\mathrm{a}_{\mathrm{k}} \cdot \mathbf{A}+$ $\mathrm{b}_{\mathrm{k}} \cdot \mathbf{B}+\mathrm{l}_{\mathrm{k}} \cdot \mathbf{L}$ 
The dependent variables in eqns. 1 and 2 are the logarithm of partition coefficients and solubility ratios for a series of solutes in a given solvent or biphasic partition system. The upper case letters denote the solute properties as follows: $\mathbf{E}$ corresponds the solute excess molar refraction descriptor in $\mathrm{cm}^{3} \mathrm{~mol}^{-1} / 10, \mathbf{S}$ is to the solute dipolarity/ polarizability descriptor, $\mathbf{A}$ and $\mathbf{B}$ represent the overall solute hydrogen bond acidity and overall solute hydrogen bond basicity descriptors, respectively, $\mathbf{V}$ is McGowan's characteristic molecular volume in $\mathrm{cm}^{3} \mathrm{~mol}^{-1} / 100$ and $\mathbf{L}$ refers to the logarithm of the gas-to-hexadecane partition coefficient measured at $298 \mathrm{~K}$. The lower case regression coefficients and constants $\left(c_{p}, e_{p}, s_{p}, a_{p}, b_{p}, v_{p}, c_{k}, e_{k}, s_{k}, a_{k}, b_{k}\right.$ and $l_{k}$ ) in Eqns. 1 and 2 are computed through a multiple linear regression analysis of experimental partition coefficient data for a specific biphasic system. Once the equation coefficients have been determined for a given biphasic partition system, further values of $\log P, \log K, \log C_{\text {so- }}$ lute,organic $/ C_{\text {solute,water }}$ and $\log C_{\text {solute,organic }} / C_{\text {solute,air }}$ can be estimated with known values for the solute descriptors. Numerical values of the solute descriptors are available for several thousand neutral organic and organometallic compounds [2, 4, 5, 30, 32, 33]. The list of available solute descriptors continues to grow as values for more complex molecules are added. Solute descriptors have also been computed for a select ions and ionic species [34-38] and for a few ion pairs [39].

We continue to publish Abraham model expressions for additional organic and ionic liquid solvents, and to revise several of our older, existing correlation equations that were based on experimental partition coefficient databases that included small numbers of solute molecules. It is important to periodically update our published $\log P$ and $\log K$ equations to take into account new experimental data. As noted in several previous papers [6, 8, 40] our published $\log P$ and $\log K$ Abraham model correlations should give reasonably accurate predictions, provided that the compound's five solute descriptors fall within the predictive area of chemical space covered by the derived Abraham model equation. The predictive area is determined by the maximum and minimum numerical values of each of the five solute descriptors in the Abraham model correlation.

In the present communication we report Abraham model correlations for the partitioning of solutes into ethylbenzene both from the gas phase (log $K$ data) and from water $(\log P$ data). As part of the present study we have updated the $\log P$ and $\log K$ correlations that we had previously reported for toluene [41]:

$\log P=0.143+0.527 \mathbf{E}-0.720 \mathbf{S}-3.010 \mathbf{A}-4.824 \mathbf{B}+$ $4.545 \mathrm{~V}$

$\left(\mathrm{N}=151 . \mathrm{SD}=0.130, \mathrm{R}^{2}=0.9968, \mathrm{~F}=4566\right)$

$\log K=0.121-0.222 \mathbf{E}+0.938 \mathbf{S}+0.467 \mathbf{A}+0.099 \mathbf{B}+$ $1.012 \mathbf{L}$

$\left(\mathrm{N}=121, \mathrm{SD}=0.111, \mathrm{R}^{2}=0.9988, \mathrm{~F}=9968\right)$
Statistical information includes: the number of solutes, $\mathrm{N}$, the correlation coefficient, $\mathrm{R}$, the standard deviation, SD, and the Fisher F-statistic, F. The databases used in constructing eqns. 3 and 4 were never published, and it is not possible to ascertain the predictive area of chemical space over which each expression is valid. The updated Abraham model $\log P$ and $\log K$ correlations presented here for toluene are based on $204 \log P(\mathrm{SD}=0.124)$ and $194 \log K(\mathrm{SD}=0.120)$ data points. Each correlation has been validated on a training set and verified with test set analyses.

\section{DATA SETS AND COMPUTATION METHODOLOGY}

Through a search of the published chemical and engineering literature [42-85] we were able to gather Raoult's law infinite dilution activity coefficient, $\gamma_{\text {solute }}{ }^{\infty}$, Henry's law constants (solute concentrations are in mole fraction), $K_{\text {Henry }}$, or solubilities for nonelectrolye organic solutes and gases dissolved in ethylbenzene and toluene. In order to apply the Abraham model, the $\gamma_{\text {solute }}{ }^{\infty}$ and $K_{\text {Henry }}$ data were converted to gas-to-organic solvent partition coefficients ( $K$ values) through standard thermodynamic expressions, Eqns. 5 and 6, as described in several of our earlier publications [2, 4-8]:

$$
\begin{aligned}
K & =\left(\frac{R T}{\gamma_{\text {solute }}{ }^{\infty} P_{\text {solute }}{ }^{o} V_{\text {solvent }}}\right) \\
K & =\left(\frac{R T}{K_{\text {Henry }} V_{\text {solvent }}}\right)
\end{aligned}
$$

or to water-to-organic solvent (or $\log P$ ) values for partition from water to solvent through Eqn. 7 , where $K_{w}$ is the solute's gas-to-water partition coefficient.

$P=K \times\left(1 / K_{w}\right) \quad$ or $\quad \log P=\log K-\log K_{w}$

The numerical constants, solute and solvent properties in Eqns. 5 and 6 are defined as follows: $\mathrm{R}$ is the universal gas constant, $\mathrm{T}$ is the system temperature, $\mathrm{P}_{\text {solute }}{ }^{\circ}$ is the vapor pressure of the solute at the temperature of the activity coefficient and/or Henry constant measurement, $T$, and $\mathrm{V}_{\text {solvent }}$ is the molar volume of the solvent. The calculation of $\log P$ from gas-to-organic solvent partition coefficient data requires knowledge of the solute's gas phase partition coefficient into water, $K_{w}$, which is available for most of the solutes being studied.

Our experimental databases also contain solubility data for several polycyclic aromatic hydrocarbons, several carboxylic acids and other miscellaneous crystalline solutes in both ethylbenzene and toluene [86-113]. The independent variable in the case of crystalline organic solutes is the molar solubility ratio, which is defined as the solute's molar solubility in the organic solvent under consideration divided by the solute's aqueous molar solubility (i.e., $C_{s o-}$ lute,organic $/ C_{\text {solute,water }}$ ) as discussed above. Molar solubilities can also be used in the $\log K$ correlation, provided that the equilibrium vapor pressure of the solute above crystalline solute, $\mathrm{P}_{\text {solute }}{ }^{\circ}$, at $298 \mathrm{~K}$ is also available. $\mathrm{P}_{\text {solute }}{ }^{\circ}$ can be 
transformed into the gas phase concentration, $C_{\text {solute,gas }}$, and the $C_{\text {solute, organid }} / C_{\text {solute, air }}$ obtained through the following equation

$\mathrm{C}_{\text {solute,organic }} / \mathrm{C}_{\text {solute,air }}=\left(\mathrm{C}_{\text {solute,organic }} / \mathrm{C}_{\text {solute,water }}\right) \times \mathrm{K}_{\mathrm{w}}$

where $K_{w}=C_{\text {solute, water }} / C_{\text {solute, air }}$. The vapor pressure, $\log K_{w}$ and aqueous solubility data needed for these calculations are reported in our previous publications.

Several published papers reported experimental partition coefficient data for substituted phenols [114-122], substituted benzenediols [123], substituted anilines [124, 125] and a few miscellaneous organic compounds [126-139]. These latter values pertain to practical partitioning studies where the aqueous and ethylbenzene (or toluene) phases were in direct contact with each other. Our previous studies $[7,8]$ have shown that the practical partition coefficients, $\log P$ values, are nearly identical to calculated solubility ratios, $C_{\text {solute, organic }} / C_{\text {solute, water, }}$, whenever the organic solvent and water are "completely" immiscible or "near completely immiscible" with each other. Given the small mole fraction solubilities of water in ethylbenzene $\left(x_{\text {water }}=2.60 \times 10^{-3}\right.$ [140]) and toluene $\left(x_{\text {water }}=2.80 \times 10^{-3}\right.$ [140]), and the small mole fraction solubilities of both aromatic hydrocarbon solvents in water $\left(x_{\text {ethylbenzene }}=3.18 \times 10^{-5}[140]\right.$ and $x_{\text {toluene }}=1.12 \times 10^{-4}[140]$ ), we elected to combine the "dry" and "wet" data sets. Water and the two aromatic hydrocarbon solvents are "almost" completely immiscible with each other at $298 \mathrm{~K}$. The experimental $\log K$ and $\log$ $P$ values at $298 \mathrm{~K}$ for ethylbenzene and toluene are listed in Tables $\mathbf{1}$ and $\mathbf{2}$, respectively. Also included in the two tables are the literature references pertaining to the $\log K$ and $\log P$ data, and the numerical values of the solute descriptors for the different compounds considered in the present study. The tabulated values are from our solute descriptor database, and were obtained using various types of experimental data, including water-to-solvent partitions, gas-to-solvent partitions, molar solubilities and chromatographic retention data $[9-11,15,16]$.

\section{RESULTS AND DISCUSSION}

We have listed in Table $\mathbf{1}$ experimental data for the partitioning for 77 solutes between the gas phase and ethylbenzene, and partitioning data for 78 solutes between water and ethylbenzene at $298 \mathrm{~K}$. The solutes considered cover a reasonably wide range of polar and nonpolar compounds, molecular sizes and shapes, and descriptor values. The experimental partition coefficient data were analyzed in accordance with the Abraham solvation parameter model to give the following two mathematical expressions:

$\log P=0.093(0.053)+0.467(0.060) \mathbf{E}-0.723(0.073) \mathbf{S}-$ 3.001(0.067) A - 4.844(0.084) B + 4.514(0.063) V

$\left(\mathrm{N}=78, \mathrm{SD}=0.137, \mathrm{R}^{2}=0.995, \mathrm{~F}=3114\right)$

and

$\log K=0.059(0.043)-0.295(0.071) \mathbf{E}+0.924(0.078) \mathbf{S}+$ $0.537(0.071) \mathbf{A}+0.098(0.088) \mathbf{B}+1.010(0.019) \mathbf{L}$
$\left(\mathrm{N}=77, \mathrm{SD}=0.143, \mathrm{R}^{2}=0.996, \mathrm{~F}=3857\right)$

All experimental data points were included in the regression analysis. Equation coefficients and statistical information were calculated using SPSS statistical software. The standard errors in the calculated coefficients are given in parenthesis after the respective equation coefficient. The statistics of both correlations are quite good as documented by the near unity values of the squared correlation coefficients and by the small standard deviations of $\mathrm{SD}=0.137$ and $\mathrm{SD}=0.143 \log$ units. The maximum deviation between the observed and predicted values was $0.40 \log$ units for both the $\log P$ (for 2,2,4-trimethylpentane) and the $\log K$ (for 2chlorophenol and pyrene) correlations. See Figs. (1 and 2) for a graphical depiction of the calculated $\log P$ and $\log K$ values based on Eqns. 9 and 10 against observed partition coefficient data. The experimental $\log P$ and $\log$ $K$ values cover ranges of about 9.5 and $12.7 \log$ units, respectively.

In order to assess the predictive ability of the two ethylbenzene correlation equations, Eqns. 9 and 10 we divided the observed data points into a training set and a test set by allowing the SPSS software to randomly select half of the experimental values. The selected data points became the training sets and the remaining half of the compounds served as the test sets. Analysis of the experimental data in the $\log P$ and $\log K$ training sets gave: $\log P=0.039(0.075)+0.492(0.100) \mathbf{E}-0.747(0.124) \mathbf{S}-$
$2.954(0.127) \mathbf{A}-4.816(0.160) \mathbf{B}+4.557(0.086) \mathbf{V}$

$\left(\mathrm{N}=39, \mathrm{SD}=0.155, \mathrm{R}^{2}=0.995, \mathrm{~F}=3114\right)$

and

$\log K=0.036(0.058)-0.367(0.048) \mathbf{E}+0.901(0.162) \mathbf{S}+$ $0.641(0.157) \mathbf{A}+0.028(0.127) \mathbf{B}+1.016(0.021) \mathbf{L}$

$\left(\mathrm{N}=39, \mathrm{SD}=0.140, \mathrm{R}^{2}=0.997, \mathrm{~F}=2048\right)$

Careful examination of Eqns 9-12 reveals that there is very little difference in the equation coefficients for the full dataset and the training dataset correlations, thus showing that both training sets of compounds are representative samples of the total $\log P$ and $\log$ $K$ data sets. The derived training set equations were then used to predict the respective partition coefficients for the compounds in the test sets. For the predicted and experimental values, we found $\mathrm{SD}=0.122$ (Eqn. 11) and $\mathrm{SD}=0.145$ (Eqn. 12), AAE (Average Absolute Error) $=$ 0.093 (Eqn. 11) and $\mathrm{AAE}=0.098$ (Eqn. 12), and $\mathrm{AE}$ (Average Error) $=-0.013$ (Eqn. 11) and $\mathrm{AE}=0.039$ (Eqn. 12). There is therefore very little bias in using Eqns. 11 and 12 with AE equal to -0.013 and $0.039 \log$ units. The training and test set analyses were performed five more times with similar results.

The toluene database considered in the present study contains 204 experimental $\log P$ values and 194 experimental $\log K$ data points. Analysis of the experimental partition 
Table 1. Experimental $\log P$ and $\log K$ Data for Solutes Dissolved in Ethylbenzene at $298 \mathrm{~K}$

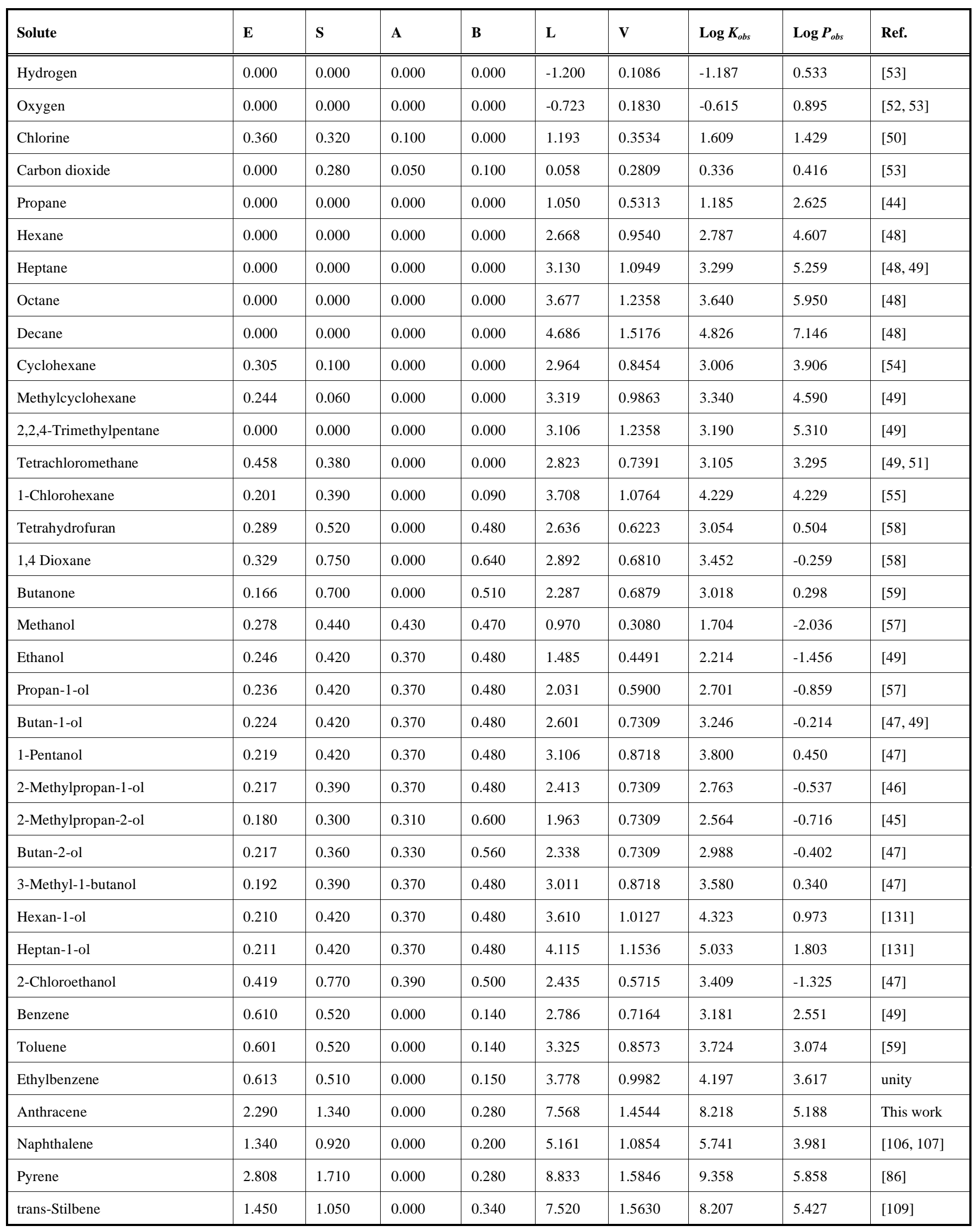


Table 1. contd....

\begin{tabular}{|c|c|c|c|c|c|c|c|c|c|}
\hline Solute & $\mathbf{E}$ & $\mathbf{S}$ & $\mathbf{A}$ & B & $\mathbf{L}$ & $\mathbf{V}$ & $\log K_{o b s}$ & $\log P_{o b s}$ & Ref. \\
\hline Ferrocene & 1.350 & 0.850 & 0.000 & 0.200 & 5.622 & 1.1209 & 6.184 & 4.264 & {$[111]$} \\
\hline Iodine & 1.398 & 0.670 & 0.280 & 0.000 & 3.681 & 0.6250 & 4.435 & 2.575 & [87] \\
\hline Phenol & 0.805 & 0.890 & 0.600 & 0.300 & 3.766 & 0.7751 & 4.947 & 0.097 & {$[122]$} \\
\hline 3-Chlorophenol & 0.909 & 1.060 & 0.690 & 0.150 & 4.773 & 0.8975 & 5.798 & 0.945 & {$[114]$} \\
\hline 4-Chlorophenol & 0.915 & 1.080 & 0.670 & 0.200 & 4.775 & 0.8975 & 6.114 & 0.954 & {$[114]$} \\
\hline 2,4-Dichlorophenol & 0.960 & 0.820 & 0.540 & 0.170 & 4.896 & 1.0199 & 5.464 & 1.814 & {$[114]$} \\
\hline 2-Methylaniline & 0.966 & 0.920 & 0.230 & 0.450 & 4.442 & 0.9571 & 5.440 & 1.380 & {$[124]$} \\
\hline 4-Methylaniline & 0.923 & 0.950 & 0.230 & 0.450 & 4.452 & 0.9571 & 5.370 & 1.280 & {$[124]$} \\
\hline 2-Nitroaniline & 1.180 & 1.370 & 0.300 & 0.360 & 5.627 & 0.9904 & 7.001 & 1.591 & {$[125]$} \\
\hline 4-Nitroaniline & 1.220 & 1.920 & 0.460 & 0.350 & 6.042 & 0.9904 & 7.925 & 0.745 & {$[125]$} \\
\hline Resorcinol & 0.980 & 1.110 & 1.090 & 0.520 & 4.618 & 0.8338 & 6.000 & -2.350 & {$[123]$} \\
\hline 1,2-Dihydroxy-4-methylbenzene & 0.950 & 1.220 & 0.850 & 0.490 & 5.135 & 0.9747 & 6.541 & -0.889 & {$[123]$} \\
\hline 4-Ethylphenol & 0.800 & 0.900 & 0.550 & 0.360 & 4.737 & 1.0569 & 5.730 & 1.230 & {$[122]$} \\
\hline Bisphenol A & 1.607 & 1.560 & 0.990 & 0.910 & 9.603 & 1.8643 & 11.499 & 0.949 & {$[133]$} \\
\hline 2,4-Dimethylphenol & 0.843 & 0.790 & 0.520 & 0.400 & 4.770 & 1.0569 & 5.531 & 1.121 & {$[121]$} \\
\hline 2,5-Dimethylphenol & 0.840 & 0.830 & 0.500 & 0.380 & 4.774 & 1.0569 & 5.660 & 1.320 & {$[116]$} \\
\hline 3,4-Dimethylphenol & 0.830 & 0.900 & 0.550 & 0.380 & 4.980 & 1.0569 & 5.910 & 1.140 & {$[121]$} \\
\hline 3,5-Dimethylphenol & 0.830 & 0.860 & 0.550 & 0.370 & 4.856 & 1.0569 & 5.841 & 1.241 & {$[121]$} \\
\hline 2-Nitrophenol & 1.015 & 1.050 & 0.050 & 0.370 & 4.760 & 0.9493 & 5.548 & 2.188 & {$[117]$} \\
\hline 3-Nitrophenol & 1.050 & 1.570 & 0.790 & 0.230 & 5.692 & 0.9493 & 7.264 & 0.204 & {$[117]$} \\
\hline 4-Nitrophenol & 1.070 & 1.720 & 0.820 & 0.260 & 5.876 & 0.9493 & 7.623 & -0.187 & {$[117]$} \\
\hline 2-Fluorophenol & 0.660 & 0.690 & 0.610 & 0.260 & 3.453 & 0.7928 & 4.206 & 0.326 & {$[115]$} \\
\hline 2-Chlorophenol & 0.853 & 0.880 & 0.320 & 0.310 & 4.178 & 0.8975 & 4.652 & 1.312 & {$[122]$} \\
\hline 2-Bromophenol & 1.037 & 0.850 & 0.350 & 0.300 & 4.802 & 0.9501 & 5.271 & 1.561 & {$[115]$} \\
\hline Propionitrile & 0.162 & 0.900 & 0.020 & 0.360 & 2.082 & 0.5451 & 3.060 & 0.240 & {$[43,51]$} \\
\hline Butylamine & 0.224 & 0.350 & 0.160 & 0.610 & 2.618 & 0.7720 & 3.014 & -0.096 & {$[56]$} \\
\hline Ethylamine & 0.236 & 0.350 & 0.160 & 0.610 & 1.677 & 0.4902 & 1.902 & -1.398 & {$[134]$} \\
\hline Propylamine & 0.225 & 0.350 & 0.160 & 0.610 & 2.141 & 0.6311 & 2.521 & -0.699 & {$[134]$} \\
\hline Butylamine (wet) & 0.224 & 0.350 & 0.160 & 0.610 & 2.618 & 0.7720 & 2.943 & -0.167 & {$[134]$} \\
\hline Diethylamine & 0.154 & 0.300 & 0.080 & 0.690 & 2.395 & 0.7720 & 2.843 & -0.147 & {$[51]$} \\
\hline Chlorobenzene & 0.718 & 0.650 & 0.000 & 0.070 & 3.657 & 0.8388 & 4.092 & 3.272 & {$[42,51]$} \\
\hline Monuron & 1.140 & 1.500 & 0.470 & 0.780 & 7.180 & 1.4768 & 8.379 & 0.749 & {$[104]$} \\
\hline Diuron & 1.280 & 1.600 & 0.570 & 0.700 & 8.060 & 1.5992 & 9.507 & 1.537 & {$[105]$} \\
\hline 2,4-Dinitrophenol & 1.200 & 1.490 & 0.090 & 0.560 & 5.981 & 1.1235 & 7.386 & 1.806 & {$[118]$} \\
\hline 1-Naphthol & 1.520 & 1.100 & 0.660 & 0.340 & 6.284 & 1.1441 & 7.592 & 1.722 & {$[129]$} \\
\hline 2-Naphthol & 1.520 & 1.080 & 0.610 & 0.400 & 6.200 & 1.1441 & 7.497 & 1.547 & {$[129]$} \\
\hline
\end{tabular}


Table 1. contd....

\begin{tabular}{|c|c|c|c|c|c|c|c|c|c|}
\hline Solute & $\mathbf{E}$ & $\mathbf{S}$ & $\mathbf{A}$ & B & $\mathbf{L}$ & $\mathbf{V}$ & $\log K_{o b s}$ & $\log P_{o b s}$ & Ref. \\
\hline 2,4-Dinitro-6-aminophenol & 1.750 & 1.850 & 0.210 & 0.800 & 7.466 & 1.2233 & 9.201 & 0.851 & {$[135]$} \\
\hline 2-Amino-6-chloro-4-nitrophenol & 1.370 & 1.210 & 0.570 & 0.700 & 6.264 & 1.1715 & 7.519 & 0.079 & {$[135]$} \\
\hline Acetylacetone & 0.412 & 0.780 & 0.000 & 0.630 & 3.326 & 0.8445 & 4.173 & 0.633 & {$[136]$} \\
\hline 2-Furaldehyde & 0.690 & 1.130 & 0.000 & 0.450 & 3.318 & 0.6962 & 4.292 & 0.462 & {$[132]$} \\
\hline 5-Methylfurfural & 0.744 & 1.110 & 0.000 & 0.520 & 3.933 & 0.8339 & 4.944 & 0.914 & {$[132]$} \\
\hline 2-Iodophenol & 1.360 & 1.000 & 0.400 & 0.350 & 4.964 & 1.0333 & & 1.772 & [115] \\
\hline
\end{tabular}

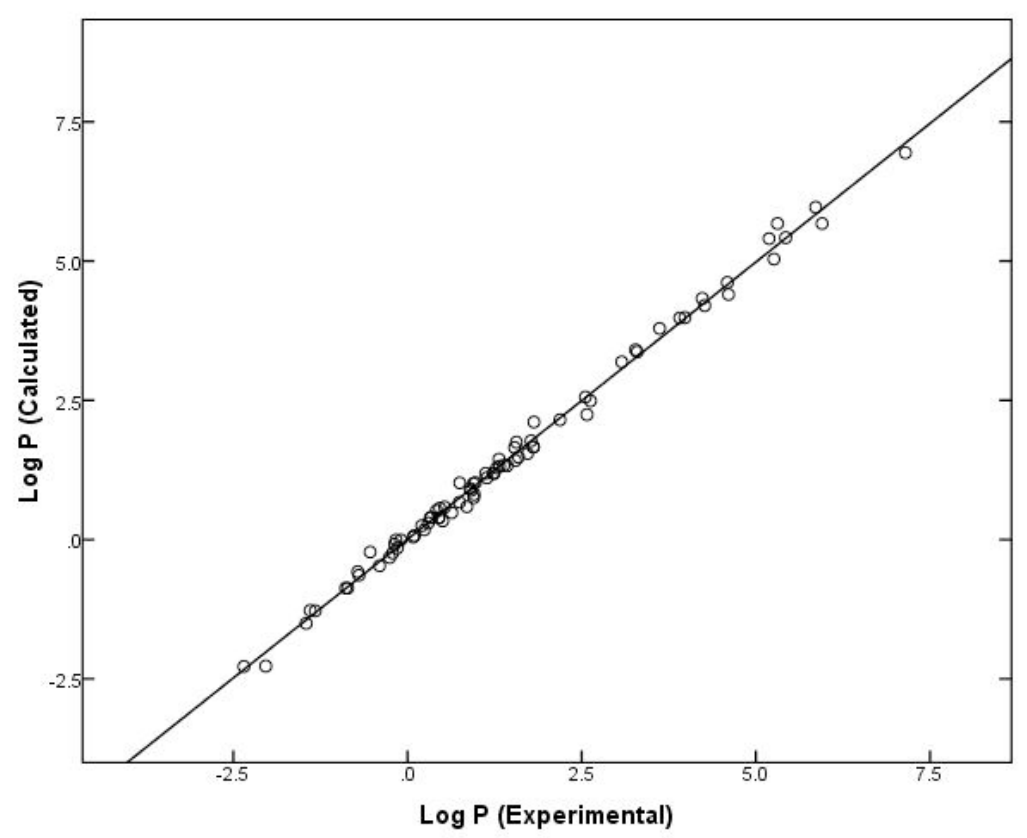

Fig. (1). Comparison of observed $\log P$ data for solutes dissolved in ethylbenzene and predicted values based on Eqn. 9.

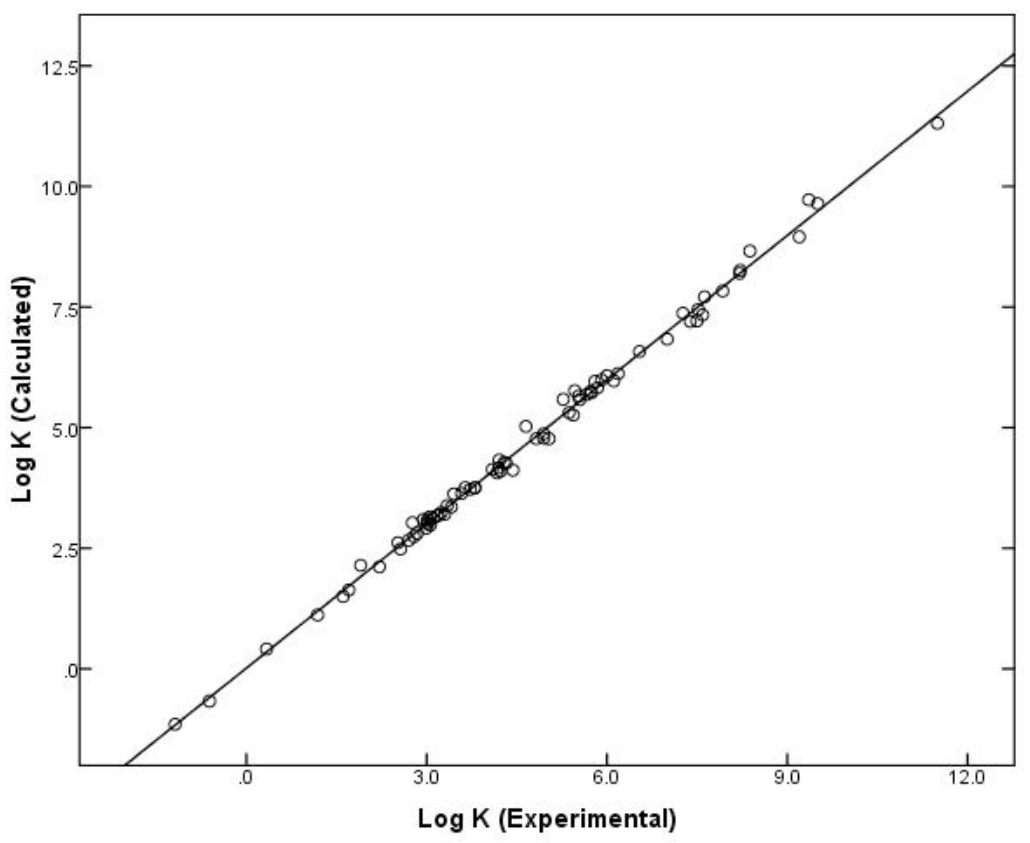

Fig. (2). Comparison of observed $\log K$ data for solutes dissolved in ethylbenzene and predicted values based on Eqn. 10. 
Table 2. Experimental $\log P$ and $\log K$ Data for Solutes Dissolved in Toluene at $298 \mathrm{~K}$

\begin{tabular}{|c|c|c|c|c|c|c|c|c|c|}
\hline Helium & 0.000 & 0.000 & 0.000 & 0.000 & -1.741 & 0.0680 & -1.650 & 0.370 & {$[60]$} \\
\hline Argon & 0.000 & 0.000 & 0.000 & 0.000 & -0.688 & 0.1900 & -0.603 & 0.867 & {$[60]$} \\
\hline Krypton & 0.000 & 0.000 & 0.000 & 0.000 & -0.211 & 0.2460 & -0.111 & 1.099 & {$[60]$} \\
\hline Hydrogen & 0.000 & 0.000 & 0.000 & 0.000 & -1.200 & 0.1086 & -1.162 & 0.558 & {$[53]$} \\
\hline Oxygen & 0.000 & 0.000 & 0.000 & 0.000 & -0.723 & 0.1830 & -0.686 & 0.824 & {$[63]$} \\
\hline Nitrogen & 0.000 & 0.000 & 0.000 & 0.000 & -0.978 & 0.2222 & -0.881 & 0.919 & {$[63]$} \\
\hline Chlorine & 0.360 & 0.320 & 0.100 & 0.000 & 1.193 & 0.3534 & 1.669 & 1.489 & {$[50]$} \\
\hline Bromine & 0.918 & 0.500 & 0.230 & 0.000 & 2.326 & 0.4586 & & 1.630 & [131] \\
\hline Methane & 0.000 & 0.000 & 0.000 & 0.000 & -0.323 & 0.2495 & -0.259 & 1.201 & {$[63]$} \\
\hline Ethane & 0.000 & 0.000 & 0.000 & 0.000 & 0.492 & 0.3904 & 0.551 & 1.891 & {$[66]$} \\
\hline Propane & 0.000 & 0.000 & 0.000 & 0.000 & 1.050 & 0.5313 & 1.177 & 2.617 & {$[62]$} \\
\hline Butane & 0.000 & 0.000 & 0.000 & 0.000 & 1.615 & 0.6722 & 1.744 & 3.264 & {$[62]$} \\
\hline 2-Methylpropane & 0.000 & 0.000 & 0.000 & 0.000 & 1.409 & 0.6722 & 1.550 & 3.250 & {$[62]$} \\
\hline Octane & 0.000 & 0.000 & 0.000 & 0.000 & 3.677 & 1.2358 & 3.853 & 5.963 & {$[61]$} \\
\hline 2,5-Dimethylhexane & 0.000 & 0.000 & 0.000 & 0.000 & 3.308 & 1.2358 & 3.476 & 5.496 & {$[61]$} \\
\hline 2,3,4-Trimethylpentane & 0.000 & 0.000 & 0.000 & 0.000 & 3.481 & 1.2358 & 3.540 & 5.420 & {$[61]$} \\
\hline Nonane & 0.000 & 0.000 & 0.000 & 0.000 & 4.182 & 1.3767 & 4.346 & 6.496 & {$[61]$} \\
\hline Cyclohexane & 0.305 & 0.100 & 0.000 & 0.000 & 2.964 & 0.8454 & 3.056 & 3.956 & {$[61]$} \\
\hline Ethylcyclohexane & 0.263 & 0.100 & 0.000 & 0.000 & 3.877 & 1.1272 & 3.918 & 5.498 & {$[61]$} \\
\hline Ethene & 0.107 & 0.100 & 0.000 & 0.070 & 0.289 & 0.3474 & 0.503 & 1.443 & {$[66]$} \\
\hline Propene & 0.100 & 0.080 & 0.000 & 0.070 & 0.946 & 0.4883 & 1.208 & 2.178 & {$[62]$} \\
\hline 1-Butene & 0.100 & 0.080 & 0.000 & 0.070 & 1.529 & 0.6292 & 1.765 & 2.775 & {$[62]$} \\
\hline cis 2-Butene & 0.140 & 0.080 & 0.000 & 0.050 & 1.737 & 0.6292 & 1.925 & 2.915 & {$[62]$} \\
\hline trans-2-Butene & 0.126 & 0.080 & 0.000 & 0.050 & 1.664 & 0.6292 & 1.871 & 2.851 & {$[62]$} \\
\hline 1-Pentene & 0.093 & 0.080 & 0.000 & 0.070 & 2.047 & 0.7700 & 2.277 & 3.507 & {$[68]$} \\
\hline Hex-1-ene & 0.080 & 0.080 & 0.000 & 0.070 & 2.572 & 0.9110 & 2.843 & 4.003 & [69] \\
\hline Isobutene (2-Methylpropene) & 0.120 & 0.080 & 0.000 & 0.080 & 1.579 & 0.6292 & 1.752 & 2.612 & {$[62]$} \\
\hline
\end{tabular}


Table 2. contd...

\begin{tabular}{|c|c|c|c|c|c|c|c|c|c|}
\hline Isopentene (3-methyl-1-butene) & 0.063 & 0.060 & 0.000 & 0.050 & 1.933 & 0.7701 & 2.149 & 3.489 & {$[68]$} \\
\hline 2-Methylbuta-1,3-diene & 0.313 & 0.230 & 0.000 & 0.100 & 2.101 & 0.7271 & 2.429 & 2.929 & {$[68]$} \\
\hline Tetrafluoromethane & -0.580 & -0.260 & 0.000 & 0.000 & -0.817 & 0.3203 & -0.799 & 1.491 & {$[63]$} \\
\hline Trichloromethane & 0.430 & 0.490 & 0.150 & 0.020 & 2.480 & 0.6167 & 3.122 & 2.332 & [68] \\
\hline Tetrachloromethane & 0.460 & 0.380 & 0.000 & 0.000 & 2.823 & 0.7391 & 3.173 & 3.363 & {$[68]$} \\
\hline Chloroethane & 0.227 & 0.400 & 0.000 & 0.100 & 1.678 & 0.5128 & 2.178 & 1.718 & {$[62]$} \\
\hline 1-Chlorohexane & 0.201 & 0.390 & 0.000 & 0.090 & 3.708 & 1.0764 & 4.306 & 4.306 & {$[55]$} \\
\hline Bromoethane & 0.370 & 0.400 & 0.000 & 0.120 & 2.120 & 0.5654 & 2.575 & 2.035 & {$[68]$} \\
\hline Iodomethane & 0.676 & 0.430 & 0.000 & 0.120 & 2.106 & 0.5077 & 2.573 & 1.923 & {$[68]$} \\
\hline Iodoethane & 0.640 & 0.400 & 0.000 & 0.150 & 2.573 & 0.6486 & 3.086 & 2.546 & {$[68]$} \\
\hline 1,1-Difluoroethane & -0.250 & 0.470 & 0.040 & 0.070 & 0.570 & 0.4258 & 1.282 & 1.202 & {$[62]$} \\
\hline Dimethyl ether & 0.000 & 0.270 & 0.000 & 0.410 & 1.285 & 0.4491 & 1.586 & 0.186 & {$[62]$} \\
\hline Diethyl ether & 0.041 & 0.250 & 0.000 & 0.450 & 2.015 & 0.7310 & 2.440 & 1.150 & [71] \\
\hline Tetrahydrofuran & 0.289 & 0.520 & 0.000 & 0.480 & 2.636 & 0.6223 & 3.218 & 0.668 & [72] \\
\hline 1,4-Dioxane & 0.329 & 0.750 & 0.000 & 0.640 & 2.892 & 0.6810 & 3.611 & -0.099 & [73] \\
\hline Butanal & 0.187 & 0.650 & 0.000 & 0.450 & 2.270 & 0.6879 & 2.935 & 0.605 & {$[81]$} \\
\hline Propanone & 0.179 & 0.700 & 0.040 & 0.490 & 1.696 & 0.5470 & 2.581 & -0.249 & {$[68]$} \\
\hline Butanone & 0.166 & 0.700 & 0.000 & 0.510 & 2.287 & 0.6879 & 3.147 & 0.427 & {$[73]$} \\
\hline Pentan-2-one & 0.143 & 0.680 & 0.000 & 0.510 & 2.755 & 0.8288 & 3.679 & 1.099 & {$[67]$} \\
\hline Hexan-2-one & 0.136 & 0.680 & 0.000 & 0.510 & 3.286 & 0.9697 & 4.105 & 1.695 & {$[67]$} \\
\hline Heptan-2-one & 0.123 & 0.680 & 0.000 & 0.510 & 3.760 & 1.1106 & 4.618 & 2.368 & {$[67]$} \\
\hline 3,3-Dimethylbutan-2-one & 0.106 & 0.620 & 0.000 & 0.510 & 2.928 & 0.9697 & 3.665 & 1.385 & {$[67]$} \\
\hline Methyl acetate & 0.142 & 0.640 & 0.000 & 0.450 & 1.911 & 0.6057 & 2.660 & 0.360 & {$[65]$} \\
\hline Ethyl acetate & 0.106 & 0.620 & 0.000 & 0.450 & 2.314 & 0.7466 & 3.164 & 1.004 & {$[68]$} \\
\hline Propyl acetate & 0.092 & 0.600 & 0.000 & 0.450 & 2.819 & 0.8875 & 3.665 & 1.615 & [67] \\
\hline Butyl acetate & 0.071 & 0.600 & 0.000 & 0.450 & 3.353 & 1.0284 & 4.083 & 2.143 & {$[67]$} \\
\hline Pentyl acetate & 0.067 & 0.600 & 0.000 & 0.450 & 3.844 & 1.1693 & 4.698 & 2.858 & [67] \\
\hline
\end{tabular}


Table 2. contd....

\begin{tabular}{|c|c|c|c|c|c|c|c|c|c|}
\hline Solute & $\mathbf{E}$ & $\mathbf{S}$ & $\mathbf{A}$ & $\mathbf{B}$ & $\mathbf{L}$ & $\mathbf{V}$ & $\log K_{o b s}$ & $\log P_{o b s}$ & Ref. \\
\hline Methyl propanoate & 0.128 & 0.600 & 0.000 & 0.450 & 2.431 & 0.7466 & 3.386 & 1.236 & {$[67]$} \\
\hline Methyl pentanoate & 0.108 & 0.600 & 0.000 & 0.450 & 3.392 & 1.0284 & 4.141 & 2.261 & {$[67]$} \\
\hline Methyl hexanoate & 0.080 & 0.600 & 0.000 & 0.450 & 3.874 & 1.1693 & 4.676 & 2.846 & {$[67]$} \\
\hline Acetonitrile & 0.237 & 0.900 & 0.070 & 0.320 & 1.739 & 0.4042 & 2.679 & -0.171 & {$[68]$} \\
\hline Propionitrile & 0.162 & 0.900 & 0.020 & 0.360 & 2.082 & 0.5450 & 3.144 & 0.324 & {$[68]$} \\
\hline Methylamine & 0.250 & 0.350 & 0.160 & 0.580 & 1.300 & 0.3493 & 1.942 & -1.398 & {$[67]$} \\
\hline n-Propylamine & 0.225 & 0.350 & 0.160 & 0.610 & 2.141 & 0.6311 & 2.580 & -0.640 & {$[65]$} \\
\hline n-Butylamine & 0.224 & 0.350 & 0.160 & 0.610 & 2.618 & 0.7720 & 3.247 & 0.137 & {$[67]$} \\
\hline Dimethylamine & 0.189 & 0.300 & 0.080 & 0.660 & 1.600 & 0.4902 & 1.964 & -1.186 & {$[67]$} \\
\hline Diethylamine & 0.154 & 0.300 & 0.080 & 0.690 & 2.395 & 0.7720 & 2.749 & -0.241 & {$[67]$} \\
\hline Dipropylamine & 0.124 & 0.300 & 0.080 & 0.690 & 3.351 & 1.0538 & 3.841 & 1.161 & {$[67]$} \\
\hline Trimethylamine & 0.140 & 0.200 & 0.000 & 0.670 & 1.620 & 0.6311 & 1.986 & -0.364 & {$[67]$} \\
\hline Triethylamine & 0.101 & 0.150 & 0.000 & 0.790 & 3.040 & 1.0538 & 3.450 & 1.090 & {$[65]$} \\
\hline Nitromethane & 0.313 & 0.950 & 0.060 & 0.310 & 1.892 & 0.4237 & 3.096 & 0.146 & {$[73]$} \\
\hline Nitroethane & 0.270 & 0.950 & 0.020 & 0.330 & 2.414 & 0.5646 & 3.388 & 0.668 & {$[75]$} \\
\hline Nitropropane & 0.242 & 0.950 & 0.000 & 0.310 & 2.894 & 0.7055 & 3.848 & 1.398 & [67] \\
\hline Water & 0.000 & 0.450 & 0.820 & 0.350 & 0.260 & 0.1673 & 1.239 & -3.401 & {$[67]$} \\
\hline Methanol & 0.278 & 0.440 & 0.430 & 0.470 & 0.970 & 0.3082 & 1.776 & -1.964 & {$[68]$} \\
\hline Ethanol & 0.246 & 0.420 & 0.370 & 0.480 & 1.485 & 0.4491 & 2.202 & -1.468 & {$[68]$} \\
\hline Propan-1-ol & 0.236 & 0.420 & 0.370 & 0.480 & 2.031 & 0.5900 & 2.746 & -0.814 & [79] \\
\hline Butan-1-ol & 0.224 & 0.420 & 0.370 & 0.480 & 2.601 & 0.7309 & 3.326 & -0.134 & [47] \\
\hline Butan-2-ol & 0.217 & 0.360 & 0.330 & 0.560 & 2.338 & 0.7309 & 3.077 & -0.313 & {$[47]$} \\
\hline 2-Methylpropan-1-ol & 0.217 & 0.390 & 0.370 & 0.480 & 2.413 & 0.7309 & 3.086 & -0.214 & {$[47]$} \\
\hline 2-Methylpropan-2-ol & 0.180 & 0.300 & 0.310 & 0.600 & 1.963 & 0.7309 & 2.739 & -0.541 & {$[80]$} \\
\hline Pentan-1-ol & 0.219 & 0.420 & 0.370 & 0.480 & 3.106 & 0.8718 & 3.860 & 0.510 & {$[65]$} \\
\hline 3-Methylbutan-1-ol & 0.192 & 0.390 & 0.370 & 0.480 & 3.011 & 0.8718 & 3.667 & 0.427 & {$[47]$} \\
\hline Hexan-1-ol & 0.210 & 0.420 & 0.370 & 0.480 & 3.610 & 1.0127 & 4.520 & 1.290 & {$[65]$} \\
\hline Heptan-1-ol & 0.211 & 0.420 & 0.370 & 0.480 & 4.115 & 1.1536 & 4.947 & 1.870 & {$[67]$} \\
\hline 2-Chloroethanol & 0.419 & 0.770 & 0.390 & 0.500 & 2.435 & 0.5715 & 3.420 & -1.180 & {$[47]$} \\
\hline Carbon disulfide & 0.876 & 0.260 & 0.000 & 0.030 & 2.370 & 0.4905 & 2.590 & 2.740 & {$[68]$} \\
\hline Sulfur hexafluoride & -0.600 & -0.200 & 0.000 & 0.000 & -0.120 & 0.4643 & -0.109 & 2.111 & {$[83]$} \\
\hline Tetramethyltin & 0.324 & 0.110 & 0.000 & 0.100 & 2.651 & 1.0431 & 2.764 & 4.294 & {$[77]$} \\
\hline Mercury & 0.850 & 0.430 & 0.000 & 0.040 & 1.721 & 0.3400 & 2.070 & 1.610 & {$[64]$} \\
\hline Benzene & 0.610 & 0.520 & 0.000 & 0.140 & 2.786 & 0.7164 & 3.268 & 2.638 & {$[68]$} \\
\hline Toluene & 0.601 & 0.520 & 0.000 & 0.140 & 3.325 & 0.8573 & 3.786 & 3.136 & Unity \\
\hline Ethylbenzene & 0.613 & 0.510 & 0.000 & 0.150 & 3.778 & 0.9982 & 4.233 & 3.653 & {$[78]$} \\
\hline o-Xylene & 0.663 & 0.560 & 0.000 & 0.160 & 3.939 & 0.9982 & 4.485 & 3.825 & {$[78]$} \\
\hline
\end{tabular}


Table 2. contd...

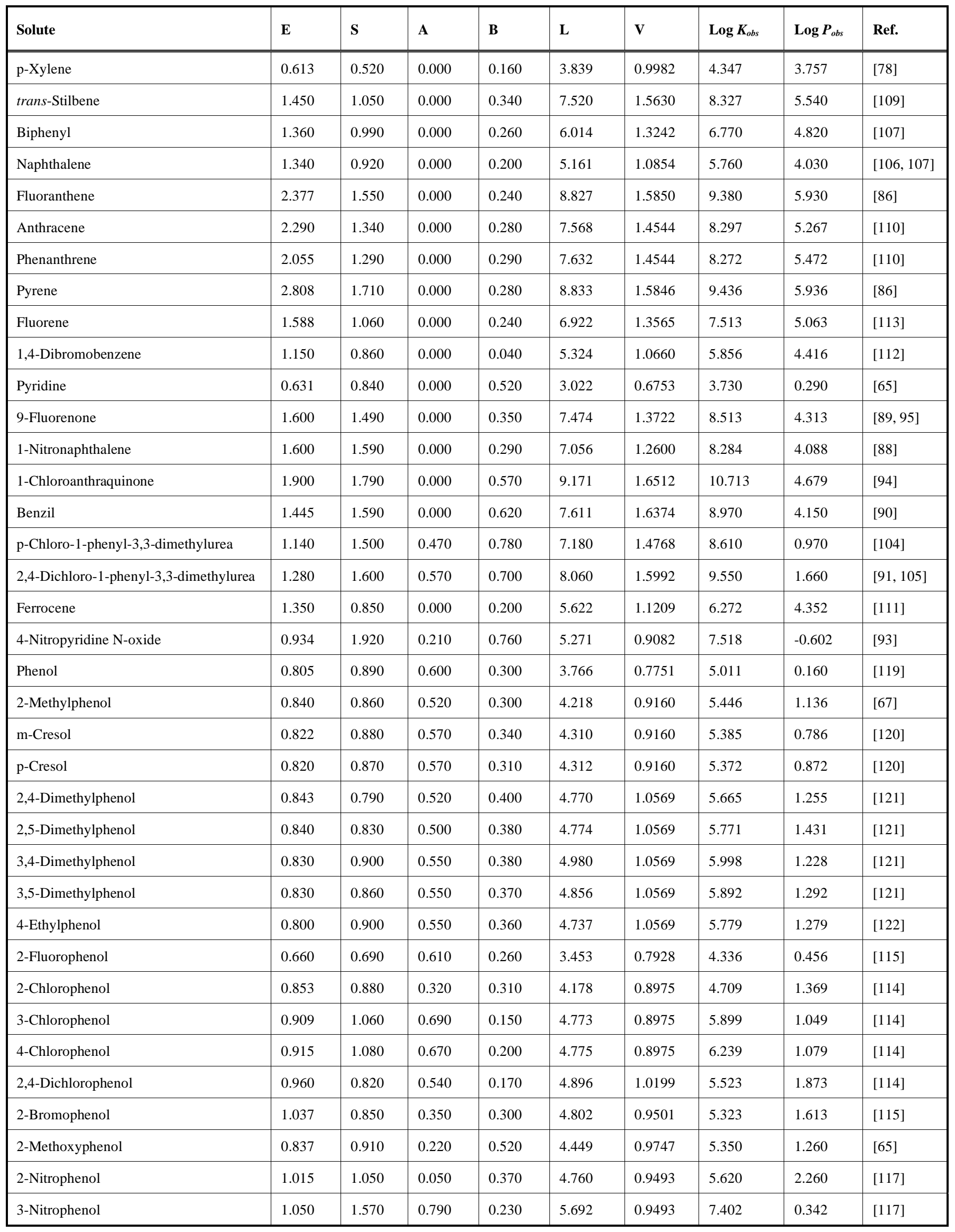


Table 2. contd....

\begin{tabular}{|c|c|c|c|c|c|c|c|c|c|}
\hline Solute & $\mathbf{E}$ & $\mathbf{S}$ & $\mathbf{A}$ & $\mathbf{B}$ & $\mathbf{L}$ & $\mathbf{V}$ & $\log K_{o b s}$ & $\log P_{o b s}$ & Ref. \\
\hline 4-Nitrophenol & 1.070 & 1.720 & 0.820 & 0.260 & 5.876 & 0.9493 & 7.750 & -0.060 & [117] \\
\hline 2,4-Dinitrophenol & 1.200 & 1.490 & 0.090 & 0.560 & 5.981 & 1.1235 & 7.520 & 1.940 & [118] \\
\hline 1-Naphthol & 1.520 & 1.100 & 0.660 & 0.340 & 6.284 & 1.1441 & 7.667 & 1.797 & [129] \\
\hline 2-Naphthol & 1.520 & 1.080 & 0.610 & 0.400 & 6.200 & 1.1441 & 7.635 & 1.685 & [129] \\
\hline 2-Nitroaniline & 1.180 & 1.370 & 0.300 & 0.360 & 5.627 & 0.9904 & 7.061 & 1.651 & {$[125]$} \\
\hline 4-Nitroaniline & 1.220 & 1.920 & 0.460 & 0.350 & 6.042 & 0.9904 & 7.993 & 0.813 & {$[125]$} \\
\hline Resorcinol & 0.980 & 1.110 & 1.090 & 0.520 & 4.618 & 0.8338 & 6.180 & -2.170 & {$[65]$} \\
\hline Aniline & 0.955 & 0.960 & 0.260 & 0.410 & 3.934 & 0.8162 & 4.970 & 0.890 & {$[65]$} \\
\hline 1-Naphthylamine & 1.670 & 1.260 & 0.200 & 0.570 & 6.490 & 1.1852 & 7.662 & 2.322 & [126] \\
\hline 2-Naphthylamine & 1.670 & 1.280 & 0.220 & 0.550 & 6.540 & 1.1852 & 7.802 & 2.332 & {$[126]$} \\
\hline Benzonitrile & 0.742 & 1.110 & 0.000 & 0.330 & 4.039 & 0.8711 & 5.283 & 2.193 & {$[85]$} \\
\hline Catechol & 0.970 & 1.100 & 0.880 & 0.470 & 4.450 & 0.8338 & 5.940 & -1.260 & {$[123]$} \\
\hline Hydroquinone & 1.063 & 1.270 & 1.060 & 0.570 & 4.827 & 0.8338 & 6.721 & -2.149 & [123] \\
\hline 1,2-Dihydroxy-4-methylbenzene & 0.950 & 1.220 & 0.850 & 0.490 & 5.135 & 0.9747 & 6.685 & -0.745 & {$[123]$} \\
\hline Thioxanthen-9-one & 1.940 & 1.441 & 0.000 & 0.557 & 8.436 & 1.5357 & 9.291 & 4.223 & {$[92]$} \\
\hline Acetone oxime & 0.390 & 0.660 & 0.370 & 0.560 & 2.557 & 0.6499 & 3.504 & -0.960 & {$[74]$} \\
\hline Cyclohexanone oxime & 0.580 & 0.900 & 0.330 & 0.610 & 4.432 & 0.9609 & 5.423 & 0.308 & {$[74]$} \\
\hline Benzoylacetone & 0.766 & 0.990 & 0.010 & 0.580 & 5.647 & 1.3114 & 6.604 & 2.914 & {$[136]$} \\
\hline 2-Methylaniline & 0.966 & 0.920 & 0.230 & 0.450 & 4.442 & 0.9571 & 5.540 & 1.480 & {$[124]$} \\
\hline 4-Methylaniline & 0.923 & 0.950 & 0.230 & 0.450 & 4.452 & 0.9571 & 5.420 & 1.330 & {$[124]$} \\
\hline Bisphenol A & 1.607 & 1.560 & 0.990 & 0.910 & 9.603 & 1.8643 & 11.626 & 1.076 & [133] \\
\hline 2-Furaldehyde & 0.690 & 1.130 & 0.000 & 0.450 & 3.318 & 0.6962 & 4.443 & 0.613 & {$[132]$} \\
\hline 5-Methylfurfural & 0.744 & 1.110 & 0.000 & 0.520 & 3.933 & 0.8339 & 5.008 & 0.978 & [132] \\
\hline 15-Crown-5 & 0.410 & 1.200 & 0.000 & 1.750 & 6.779 & 1.7025 & 8.250 & -1.150 & {$[127]$} \\
\hline 16-Crown-5 & 0.410 & 1.170 & 0.000 & 1.760 & 7.276 & 1.8434 & 8.580 & -0.600 & {$[127]$} \\
\hline Benzo 15-Crown-5 & 1.055 & 1.940 & 0.000 & 1.590 & 9.403 & 2.0285 & 11.470 & 0.920 & [127] \\
\hline 18-Crown-6 & 0.410 & 1.470 & 0.000 & 2.100 & 8.228 & 2.0430 & 9.840 & -1.590 & {$[127]$} \\
\hline Dibenzo-18-Crown-6 & 1.690 & 2.730 & 0.000 & 1.780 & 13.384 & 2.6950 & 16.150 & 2.770 & {$[127]$} \\
\hline Dibenzo-24-Crown-8 & 1.680 & 3.400 & 0.000 & 2.340 & 16.414 & 3.3760 & 20.310 & 2.930 & {$[127]$} \\
\hline AC-Benzo-18-Crown-6 ${ }^{a}$ & 0.684 & 2.650 & 0.000 & 1.850 & 11.100 & 2.4776 & 14.357 & 1.307 & {$[128]$} \\
\hline 3-Chlorobenzoic acid & 0.840 & 0.950 & 0.630 & 0.320 & 5.197 & 1.0541 & 6.270 & 1.120 & {$[96]$} \\
\hline 4-Chlorobenzoic acid & 0.840 & 1.020 & 0.630 & 0.270 & 4.947 & 1.0540 & 5.960 & 1.260 & {$[102]$} \\
\hline 3-Nitrobenzoic acid & 0.990 & 1.130 & 0.730 & 0.530 & 5.535 & 1.1059 & 7.020 & 0.138 & {$[98]$} \\
\hline 4-Nitrobenzoic acid & 0.990 & 1.520 & 0.680 & 0.400 & 5.770 & 1.1059 & 7.405 & 0.620 & [99] \\
\hline 2-Methylbenzoic acid & 0.730 & 0.840 & 0.420 & 0.440 & 4.677 & 1.0726 & 5.530 & 1.230 & [100] \\
\hline 2-Methyoxybenzoic acid & 0.899 & 1.410 & 0.450 & 0.620 & 5.636 & 1.1313 & 7.250 & 0.398 & [97] \\
\hline 4-Methoxybenzoic acid & 0.899 & 1.250 & 0.620 & 0.520 & 5.741 & 1.1313 & 7.240 & 0.484 & {$[97]$} \\
\hline
\end{tabular}


Table 2. contd....

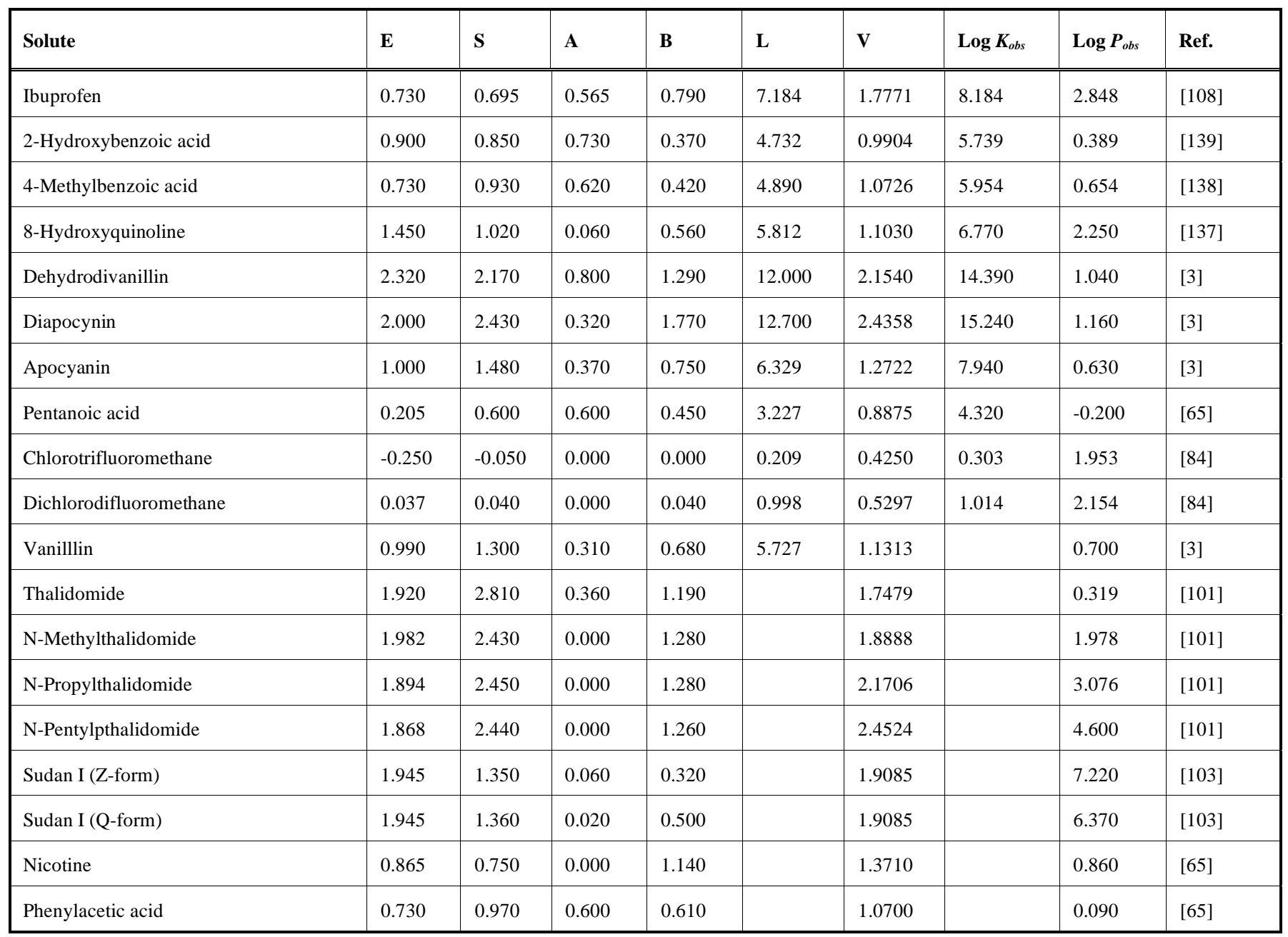

a 1,2-bis[2-(2-methoxyethoxy)ethoxy]benzene.

coefficient values tabulated in Table 2 yielded the following two Abraham model expressions:

$\log P=0.125(0.021)+0.431(0.030) \mathbf{E}-0.644(0.036) \mathbf{S}-$ 3.002(0.036) A - 4.748(0.041) B + 4.524(0.033) V

$\left(\mathrm{N}=204, \mathrm{SD}=0.124, \mathrm{R}^{2}=0.996, \mathrm{~F}=8965\right)$

and

$\log K=0.085(0.015)-0.400(0.034) \mathbf{E}+1.063(0.039) \mathbf{S}+$ $0.501(0.035) \mathbf{A}+0.154(0.041) \mathbf{B}+1.011(0.009) \mathbf{L}$

$\left(\mathrm{N}=194, \mathrm{SD}=0.120, \mathrm{R}^{2}=0.999, \mathrm{~F}=27911\right)$

Both correlations provide a reasonably accurate mathematical description of the experimental partition coefficient data as graphically shown in Figs. (3 and 4$)$ for datasets that span ranges of $10.6 \log$ units $(\log P)$ and $22.0 \log$ units $(\log$ $K)$, respectively. The maximum deviation between the observed and predicted values was $0.35 \log$ units for the $\log P$ (for 3,3-dimethylbutan-2-one) and $0.41 \log$ units for the $\log$ $K$ (for 2-chlorophenol and fluoranthene) correlations. As noted in the introduction, Abraham model $\log P$ and $\log K$ correlations were derived previously for toluene. The earlier correlations were based on much smaller data sets. In the log $K$ data set, we were able to increase the number of experimental values by more than $50 \%$, from $\mathrm{N}=121$ (Eqn. 4) to $\mathrm{N}=194$ (Eqn. 14).

Equations 13 and 14 were validated as before by allowing the SPSS software to randomly divide the total data points into training and test sets as before. Analyses of the experimental data in the two training sets yielded:

$\log P=0.093(0.027)+0.408(0.039) \mathbf{E}-0.652(0.047) \mathbf{S}-$ 2.981(0.048) A - 4.789(0.054) B + 4.596(0.042) V

$\left(\mathrm{N}=102, \mathrm{SD}=0.113, \mathrm{R}^{2}=0.997, \mathrm{~F}=5762\right)$

and

$\log K=0.084(0.020)-0.427(0.054) \mathbf{E}+1.104(0.064) \mathbf{S}+$ $0.450(0.057) \mathbf{A}+0.174(0.062) \mathbf{B}+1.005(0.012) \mathbf{L}$

$\left(\mathrm{N}=97, \mathrm{SD}=0.118, \mathrm{R}^{2}=0.999, \mathrm{~F}=13844\right)$

Careful examination of Eqns. 13 versus 15, and Eqns. 14 versus 16 reveals that there is very little difference in the equation coefficients for the full dataset and the training 


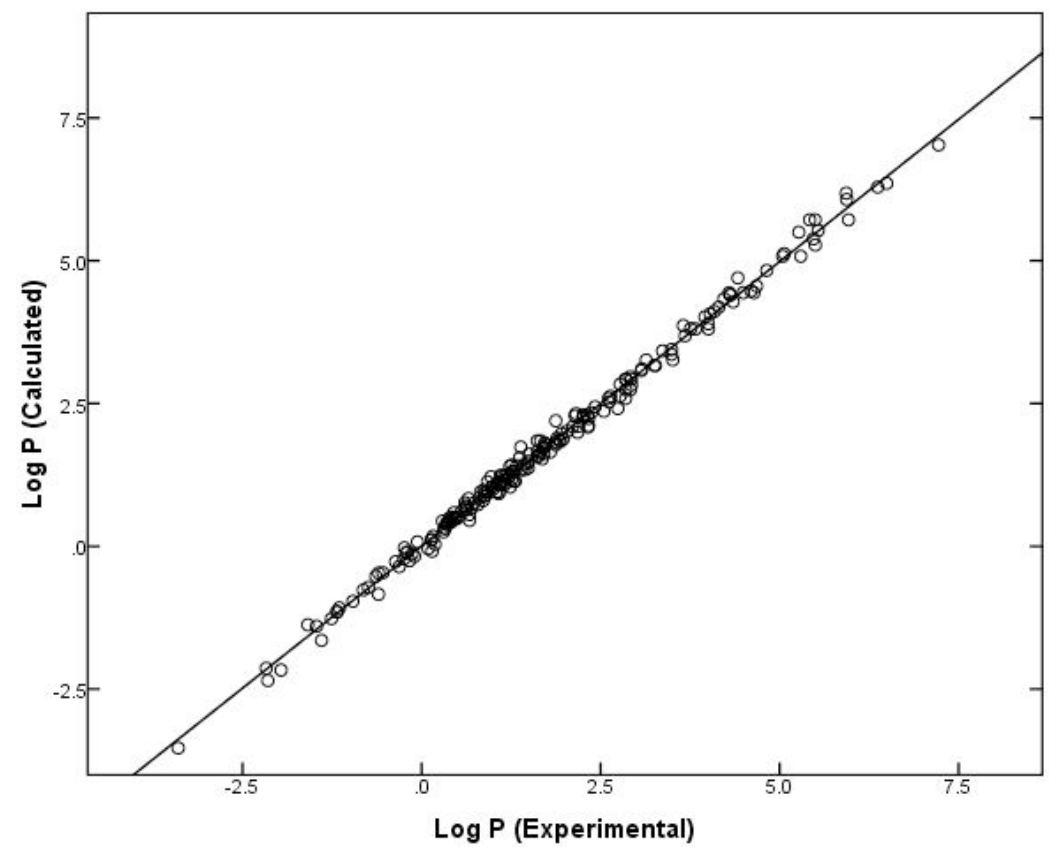

Fig. (3). Comparison of observed $\log P$ data for solutes dissolved in toluene and predicted values based on Eqn. 13.

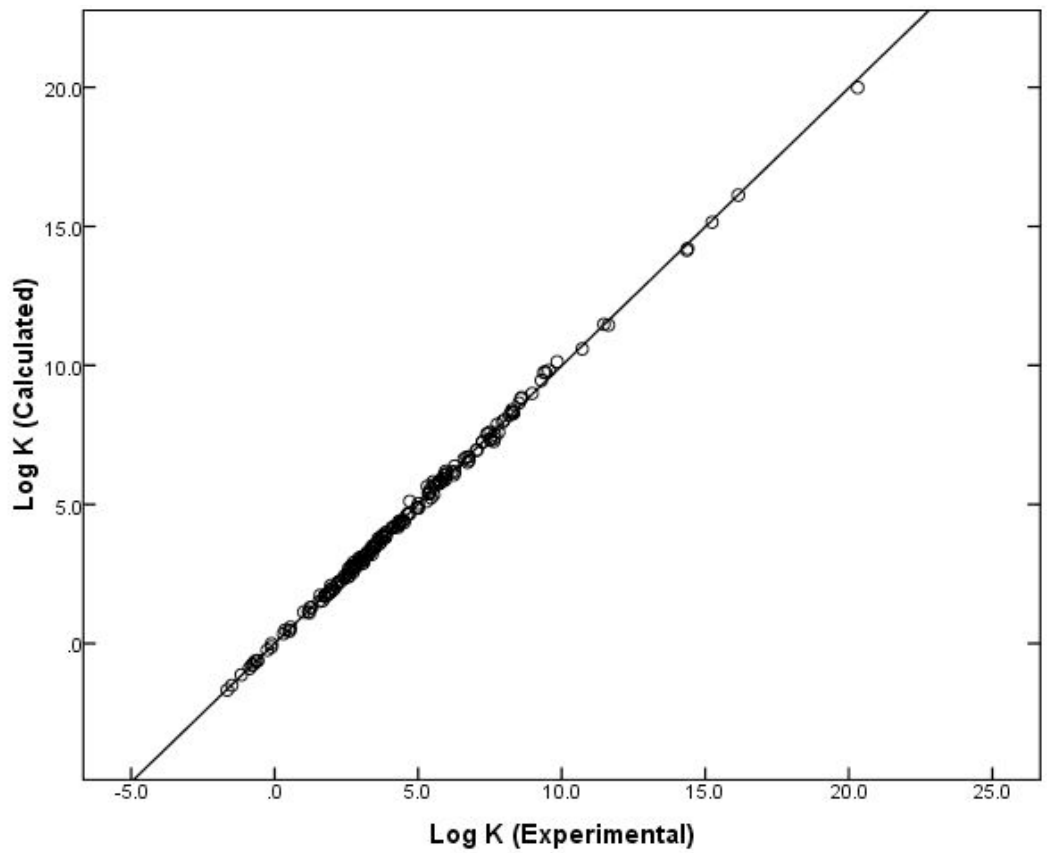

Fig. (4). Comparison of observed $\log K$ data for solutes dissolved in toluene and predicted values based on Eqn. 14.

dataset correlations. Both training sets of compounds are representative samples of the total $\log P$ and $\log K$ data sets. The derived training set equations were then utilized to estimate the respective partition coefficients for the compounds in the test sets. For the estimated and experimental values, we found $\mathrm{SD}=0.138$ (Eqn. 15) and $\mathrm{SD}=0.125$ (Eqn. 16), $\mathrm{AAE}=0.102$ (Eqn. 15) and $\mathrm{AAE}=0.092$ (Eqn. 16), and $\mathrm{AE}$ $=-0.008$ (Eqn. 15) and $\mathrm{AE}=0.025$ (Eqn. 16). There is therefore very little bias in using Eqns. 15 and 16 with $\mathrm{AE}$ equal to -0.008 and $0.025 \log$ units.
The present study documents that the correlations based on the Abraham solvation parameter model provide reasonably accurate mathematical descriptions of solute transfer at $298 \mathrm{~K}$ from both water and from the gas phase into both ethylbenzene and toluene. The derived correlations should provide reasonably accurate estimations of the water-toethylbenzene, water-to-toluene, gas-to-ethylbenzene and gas-to-toluene partition coefficients at $298 \mathrm{~K}$ for additional organic compounds and gases, provided that each of the compound's solute descriptors falls within the range of predictive chemical space defined by the respective correlation 
equation. The estimated partition coefficients can be converted to infinite dilution activity coefficients, $\gamma_{\text {solute }}{ }^{\infty}$, through the standard thermodynamic relationships given by Eqns. 5 - 7. Partition coefficients calculated using Eqns. 9, 10,13 and 14 pertain to $298 \mathrm{~K}$. Standard thermodynamic relationships enable the predicted $\log K$ and $\log P$ values to be extrapolated to other temperatures [141]. In this regard, Mintz et al. published enthalpy of solvation correlations,

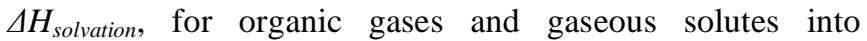
water [141] and toluene [142]. The published $\Delta H_{\text {solvation }}$ correlations allow one to extrapolate predicted water-totoluene and gas-to-toluene partition coefficients based on Eqs. 13 and 14 to other temperatures not too far removed from $298 \mathrm{~K}$.

\section{CONFLICT OF INTEREST}

None declared.

\section{ACKNOWLEDGEMENTS}

Matthew Loera thanks the National Science Foundation for support received under NSF-REU grant (CHE-1004878). Vicky Chou, Amanda Quay and Connie Shen thank the University of North Texas's Texas Academy of Math and Science (TAMS) program for a summer research award.

\section{NOMENCLATURE}

\section{English Symbols}

a $=$ Solvent coefficient in the Abraham model representing hydrogen-bond basicity of the solvent

$\mathbf{A}$

$=$ Abraham model solute descriptor representing hydrogen-bond acidity
AAE $=$ Absolute average error
$\mathrm{AE}=$ Absolute error
b $=$ Solvent coefficient in the Abraham model representing hydrogen-bond acidity of the solvent

B $=$ Abraham model solute descriptor representing hydrogen-bond basicity

c $=$ Constant in the Abraham model correlation equation

C $=$ Concentration of the solute expressed in units of moles per liter

e $\quad=$ Solvent equation coefficient in the Abraham model measuring the solvent's interaction with $\pi$ - and nonbonding electrons on the solute

E $\quad=$ Abraham model solute descriptor describing excess molar refraction

$\mathrm{F} \quad=$ Fisher F-statistic

$K=$ Gas-to-liquid partition coefficient of the solute

$K_{\text {Henry }}=$ Henry's constant of the solute in the given solvent
$K_{w} \quad=\quad$ Gas-to-water partition coefficient of the solute
$1=$ Solvent equation coefficient in the Abraham model describing dispersion forces
$\mathbf{L}=$ Abraham model solute descriptor defined as the logarithm of the solute's gas-to- hexadecane partition coefficient at $298 \mathrm{~K}$
$\mathrm{N} \quad=\quad$ Number of experimental data points
$P \quad=\quad$ Water-to-solvent partition coefficient
$\mathrm{P}_{\text {solute }}{ }^{\mathrm{o}}=$ Vapor pressure of the solute at temperature of the activity coefficient measurement
$\mathrm{R}=$ Correlation coefficient for the derived corre- lation model, also used as the gas constant in Eqns. 5 and 6
$\mathrm{s}=$ Solvent equation coefficient in the Abraham model measuring dipolarity/polarizability of the solvent
$\mathbf{S}=$ Abraham model solute descriptor describing polarity/polarizability
SD $=$ Standard deviation
$T \quad=$ Temperature
$\mathrm{v}=$ Solvent equation coefficient in the Abraham model
$\mathbf{V}=$ McGowan volume of the solute
$\mathrm{V}_{\text {solvent }}=$ Molar volume of the solvent
$x \quad=$ Mole fraction

\section{Greek symbols}

$\gamma_{\text {solute }}^{\infty}=$ Infinite dilution activity coefficient of solute

$\Delta H_{\text {solvation }}=$ Enthalpy of solvation of the solute in the given solvent

\section{REFERENCES}

[1] M. H. Abraham and W. E. Acree, Jr., "The transfer of neutral molecules, ions and ionic species from water to ethylene glycol and to propylene carbonate; descriptors for pyridinium cations," New J. Chem., vol. 34, pp. 2298-2305, 2010.

[2] L. M. Grubbs, M. Saifullah, N. E. De La Rosa, S. Ye, S. S. Achi, W. E. Acree, Jr. and M. H. Abraham, "Mathematical correlations for describing solute transfer into functionalized alkane solvents containing hydroxyl, ether, ester or ketone solvents," Fluid Phase Equilibr., vol. 298, pp. 48-53, 2010.

[3] M. H. Abraham, R. E. Smith, R. Luchtefeld, A. J. Boorem, R. Luo and W. E. Acree, Jr., "Prediction of solubility of drugs and other compounds in organic solvents," J. Pharm. Sci., vol. 99, pp. 1500$1515,2010$.

[4] L. M. Sprunger, S. S. Achi, R. Pointer, W. E. Acree, Jr. and M. H. Abraham, "Development of Abraham model correlations for solvation characteristics of secondary and branched alcohols," Fluid Phase Equilibr., vol. 288, pp. 121-127, 2010.

[5] L. M. Sprunger, S. S. Achi, R. Pointer, B. H. Blake-Taylor, W. E. Acree, Jr. and M. H. Abraham, "Development of Abraham model correlations for solvation characteristics of linear alcohols," Fluid Phase Equilibr., vol. 286, no. 2, pp. 170-174, 2009.

[6] T. W. Stephens, N. E. De La Rosa, M. Saifullah, S. Ye, V. Chou, A. N. Quay, W. E. Acree Jr. and M. H. Abraham, "Abraham model correlations for transfer of neutral molecules and ions to sulfolane," Fluid Phase Equilibr., vol. 309, pp. 30-35, 2011. 
[7] T. W. Stephens, N. E. De La Rosa, M. Saifullah, S. Ye, V. Chou, A. N. Quay, W. E. Acree Jr. and M. H. Abraham, "Abraham model correlations for solute partitioning into $o$-xylene, $m$-xylene and $p$ xylene from both water and the gas phase," Fluid Phase Equilibr., vol. 308, pp. 64-71, 2011.

[8] L. M. Sprunger, S. S. Achi, W. E. Acree, Jr., M. H. Abraham, A. J. Leo and D. Hoekman, Correlation and prediction of solute transfer to chloroalkanes from both water and the gas phase, Fluid Phase Equilibr., vol. 281, pp. 144-162, 2010.

[9] L. M. Grubbs, M. Saifullah, N. E. De La Rosa, W. E. Acree, Jr., M. H. Abraham, Q. Zhao and J. L. Anderson,Cation-specific and Anion-specific Abraham Model Correlations for Solute Transfer into Ionic Liquids, Glob. J. Phys. Chem., vol. 1, pp. 1-19, 2010.

[10] U. Domanska, M. Królikowska, W. E. Acree, Jr. and G. A. Baker, "Activity coefficients at infinite dilution measurements for organic solutes and water in the ionic liquid 1-ethyl-3-methylimidazolium tetracyanoborate," J. Chem. Thermodyn., vol. 43, pp. 1050-1057, 2011.

[11] L. M. Grubbs, S. Ye, M. Saifullah, M. C. McMillan-Wiggins, W. E. Acree, Jr., M. H. Abraham, P. Twu and J. L. Anderson, "Correlations for describing gas-to-ionic liquid partitioning at $323 \mathrm{~K}$ based on ion-specific equation coefficient and group contribution versions of the Abraham model," Fluid Phase Equilibr., vol. 301, pp. 257-266, 2011.

[12] A.-L. Revelli, F. Mutelet, J.-N. Jaubert, M. Garcia-Martinez, L. M. Sprunger, W. E. Acree, Jr. and G. A. Baker, "Study of ether-, alcohol-, or cyano-functionalized ionic liquids using inverse gas chromatography," J. Chem. Eng. Data, vol. 55, pp. 2434-2443, 2010.

[13] J.-C. Moise, F. Mutelet, J.-N. Jaubert, L. M. Grubbs, W. E. Acree, Jr. and G. A. Baker, "Activity coefficients at infinite dilution of organic compounds in four new imidazolium-based ionic liquids," $J$. Chem. Eng. Data, vol. 56, pp. 3106-3114, 2011.

[14] F. Mutelet, A.-L. Revelli, J.-N. Jaubert, L.M. Sprunger, W. E. Acree, Jr. and G. A. Baker, "Partition coefficients of organic compounds in new imidazolium and tetralkylammonium based ionic liquids using inverse gas chromatography," J. Chem. Eng. Data, vol. 55, pp. 234-242, 2010.

[15] A.-L. Revelli, L. M. Sprunger, J. Gibbs, W. E. Acree, Jr., G. A. Baker and F. Mutelet, "Activity coefficients at infinite dilution of organic compounds in trihexyl(tetradecyl)-phosphonium bis(trifluoromethylsulfonyl)imide using inverse gas chromatography," J. Chem. Eng. Data, vol. 54, pp. 977-985, 2009.

[16] A. Geisler, S. Endo, K.-U. Goss, "Partitioning of polar and non-polar neutral organic chemicals into human and cow milk," Environ. Int., vol. 37, pp. 1253-1258, 2011.

[17] L. M. Sprunger, A. Proctor, W. E. Acree, Jr. and M. H. Abraham, "Characterization of the sorption of gaseous and organic solutes onto polydimethyl siloxane solid-phase microextraction surfaces using the Abraham model," J. Chromatogr., A, vol. 1175, pp. 162173, 2007

[18] H. P. H. Arp, S. Endo, K.-U. Goss, "Comment on assessment of PDMS-water partition coefficients: implications for passive environmental sampling of hydrophobic compounds," Environ. Sci. Technol., vol. 44, pp. 8787-8788, 2010.

[19] S. Endo, S. E. Hale, K.-U. Goss and P. H. Arp, "Equilibrium partition coefficients of diverse polar and nonpolar organic compounds to polyoxymethylene (POM) passive sampling devices," Environ. Sci. Technol., vol. 45, pp. 10124-10132, 2011..

[20] S. Endo, S. T. J. Droge and K.-U. Goss, "Polyparameter linear free energy models for polyacrylate fiber-water partition coefficients to evaluate the efficiency of solid-phase microextraction," Analyt. Chem., vol. 83, pp.1394-1400, 2011.

[21] T. Karunasekara and C. F. Poole, "Models for liquid-liquid partition in the system ethylene glycol-organic solvent and their use for estimating descriptors for organic compounds," Chromatographia, vol. 73, pp. 941-951, 2011.

[22] T. Karunasekara and C. F. Poole, "Models for liquid-liquid partition in the system formamide-organic solvent and their use for estimating descriptors for organic compounds," Talanta, vol. 83, pp. 1118-1125, 2011.

[23] T. Karunasekara and C. F. Poole, "Models for liquid-liquid partition in the system propylene carbonate-organic solvent and their use for estimating descriptors for organic compounds," J. Chromatogr., A, vol. 1218, pp. 809-816, 2011.
[24] T. Karunasekara and C. F. Poole, "Model for the partition of neutral compounds between n-heptane and formamide," J. Sep. Sci., vol. 33, pp. 1167-1173, 2010.

[25] J. Qian and C. F. Poole, "Distribution of neutral organic compounds between n-heptane and fluorine-containing alcohols," $J$. Chromatogr., A, vol. 1143, no. 1-2, pp. 276-283, 2007.

[26] H. Ahmed, C. F. Poole and G. E. Kozerski, "Determination of descriptors for organosilicon compounds by gas chromatography and non-aqueous liquid-liquid partitioning," J. Chromatogr., A, vol. 1169, pp. 179-192, 2007.

[27] H. Ahmed and C. F. Poole, "Distribution of neutral organic compounds between n-heptane and methanol or N,Ndimethylformamide," J. Sep. Sci., vol. 29, pp. 2158-2165, 2006.

[28] H. Ahmed and C. F. Poole, "Model for the distribution of neutral organic compounds between n-hexane and acetonitrile," $J$. Chromatogr., A, vol. 1104, pp. 82-90, 2006.

[29] T. Karunasekara and C. F. Poole, "Models for liquid-liquid partition in the system dimethyl sulfoxide-organic solvent and their use for estimating descriptors for organic compounds," $J$. Chromatogr., A, vol. 1218, pp. 4525-4536, 2011.

[30] M. H. Abraham, "Scales of solute hydrogen-bonding: their construction and application to physicochemical and biochemical processes," Chem. Soc. Rev., vol. 22, pp. 73-83, 1993.

[31] M. H. Abraham, A. Ibrahim and A. M. Zissimos, "Determination of sets of solute descriptors from chromatographic measurements," J. Chromatogr. A, vol. 1037, pp. 29-47, 2004.

[32] M. H. Abraham, A. Ibrahim, Y. Zhao and W. E. Acree, Jr., "A data base for partition of volatile organic compounds and drugs from blood/plasma/serum to brain, and an LFER analysis of the data," $J$ Pharm. Sci., vol. 95, pp. 2091-2100, 2006

[33] M. H. Abraham, H. S. Chadha, G. S. Whiting and R. C. Mitchell, "Hydrogen bonding. 32. An analysis of water-octanol and wateralkane partitioning and the $\Delta \log$ P parameter of Seiler," J. Pharm. Sci., vol. 83, pp. 1085-1100, 1994

[34] M. H. Abraham and W. E. Acree, Jr., "Equations for the transfer of neutral molecules and ionic species from water to organic phases," J. Org. Chem., vol. 75, pp. 1006-1015, 2010.

[35] M. H. Abraham and W. E. Acree, Jr., "The transfer of neutral molecules, ions and ionic species from water to wet octanol," Phys. Chem. Chem. Phys., vol. 12, pp. 13182-13188, 2010.

[36] M. H. Abraham and W. E. Acree, Jr., "Solute descriptors for phenoxide anions and their use to establish correlations of rates of reaction of anions with iodomethane," J. Org. Chem., vol. 75, pp. 3021-3026, 2010.

[37] K. Zhang, M. Chen, G. K. E. Scriba, M. H. Abraham, A. Fahr and X. Liu, "Linear free energy relationship analysis of retention factors in cerasome electrokinetic chromatography intended for predicting drug skin permeation," J. Pharm. Sci., vol. 100, pp. 3105-3113, 2011.

[38] M. H. Abraham, "The permeation of neutral molecules, ions, and ionic species through membranes: Brain permeation as an example," J. Pharm. Sci., vol. 100, pp. 1690-1701, 2011.

[39] M. H. Abraham and W. E. Acree, Jr., "Hydrogen bond descriptors and other properties of ion pairs," N. J. Chem., vol. 35, pp. 17401750, 2011.

[40] L. M. Sprunger, J. Gibbs, A. Proctor, W. E. Acree, Jr., M. H. Abraham, Y. Meng, C. Yao and J. L. Anderson, "Linear free energy relationship correlations for room temperature ionic liquids: revised cation-specific and anion-specific equation coefficients for predictive applications covering a much larger area of chemical space," Ind. Eng. Chem. Res., vol. 48, pp. 4145-4154, 2009.

[41] M. H. Abraham, C. E. Green, W. E. Acree, Jr., C. E. Hernandez and L. E. Roy, "Descriptors for solutes from the solubility of solids: trans-stilbene as an example," J. Chem. Soc., Perkin Trans. 2, pp. 2677-2682, 1998.

[42] H. C. Van Ness and B. D. Smith, "Excess Gibbs energy. Chlorobenzene-ethylbenzene system," Int. DATA Ser., Sel. Data Mix., Ser. A, no. 1, p. 24, 1982 .

[43] H. C. Van Ness and B. D. Smith, "Excess Gibbs energy. Propanenitrile-ethylbenzene system," Int. DATA Ser., Sel. Data Mix. Ser. A, no. 1, p. 22, 1982.

[44] W. Hayduk, Propane, Butane and 2-Methylpropane, IUPAC Solubility Data Series, vol. 24, pp. 218-250, 1986.

[45] P. Oracz, "Excess Gibbs energy. 2-Methyl-2-propanolethylbenzene system," Int. DATA Ser., Sel. Data Mix., Ser. A, p. $249,1989$. 
[46] P. Oracz, P., "Excess Gibbs energy. 2-Methyl-1-propanol - ethylbenzene system," Int. DATA Ser., Sel. Data Mix., Ser. A, p. 237, 1989.

[47] A. Ignat and L. Molder, "Activity coefficients of alcohols in organic solvents and water at infinite dilution," Eesti NSV Teaduste Akadeemia Toimetised, Keemia, vol. 38, pp. 11-16, 1989.

[48] M. Góral, "Vapor-liquid equilibria in non-polar mixtures. III. Binary mixtures of alkylbenzenes and n-alkanes at $313.15 \mathrm{~K}$," Fluid Phase Equilibr., vol. 102, pp. 275-286, 1994.

[49] S. Shen, S. Wang, J. Zhang and J. Shi, "Determination and application of activity coefficients at infinite dilution," J. Chem. Ind. Eng. (China), vol. 39, pp. 501-507, 1988.

[50] M. Lohse and W. D. Deckwer, "Solubility of chlorine in benzene, toluene, ethylbenzene, $o_{-}, m_{-}$, and $p$-xylenes, and 2-, 3-, and 4chlorotoluenes," J. Chem. Eng. Data, vol. 26, pp. 159-161, 1981.

[51] J. Gmehling, U. Onken and W. Arlt, Vapor-Liquid Equilibrium Data Collection; Chemical Data Series, Volume I, parts 2b-d, DECHEMA, Franfurt/Main, Germany, 1978-1984.

[52] N. K. Naumenko, N. N. Mukhin and V. B. Aleskovskii, "Solubility of oxygen in liquid aromatic compounds," Zh. Priklad. Khim., vol. 42, pp. 2522-2528, 1969.

[53] P. Lühring and A. Schumpe, "Gas solubilities (hydrogen, helium, nitrogen, carbon monoxide, oxygen, argon, carbon dioxide) in organic liquids at 293.2 K," J. Chem. Eng. Data, vol. 34, no. 2, pp. 250-252, 1989.

[54] D. V. S. Jain and O. P. Yadav, "Vapor pressures and excess Gibbs energies for the binary mixtures of ethylbenzene with cyclohexane and carbon tetrachloride," Indian J. Chem., vol. 12, pp. 718-720, 1974.

[55] H. I. Paul, J. Krug and H. Knapp, "Measurements of VLE, ${ }^{E}$ and $\mathrm{h}^{\mathrm{E}}$ for binary mixtures of 1-chlorohexane with three nalkylbenzenes: toluene, ethylbenzene, n-propylbenzene," J. Chem. Eng. Data, vol. 33, pp. 453-460, 1988.

[56] J. Fernandez, R. Garriga, I. Velasco and S. Otin, "Thermodynamic properties of binary mixtures containing $\mathrm{n}$-alkylamines II. Isothermal vapour-liquid equilibrium and excess molar enthalpy of nalkylamine + ethylbenzene mixtures. Measurement and analysis in terms of group contributions," Fluid Phase Equilibr., vol. 163, pp. 231-242, 1999.

[57] P. Vrbka and V. Dohnal, "Limiting activity coefficients in binary mixtures of 1-alkanols and ethylbenzene," Fluid Phase Equilibr., vol. 263, pp. 64-70, 2008.

[58] D. V. S. Jain and R. S. Sidhu, "Excess molar Gibbs free energies of methylcyclohexane $+1,4$-dioxane and + tetrahydrofuran and of ethylbenzene +1,4-dioxane and + tetrahydrofuran at 303.15 and 323.15 K," J. Chem. Thermodyn., vol. 16, pp. 111-114, 1984.

[59] H. Arm, F. Hugli and R. Signer, "Thermodynamic measurements of mixtures of ethylbenzene with toluene and methyl ethyl ketone," Helv. Chim. Acta, vol. 40, pp. 1200-1205, 1957.

[60] J. H. Saylor and R. Battino, "The solubilities of the rare gases in some simple benzene derivatives," J. Phys. Chem., vol. 62, pp. 1334-1337, 1958.

[61] D. I. Eikens, "Applicability of theoretical and semi-empirical models for predicting infinite dilution activity coefficients. (Volumes I and II)," Ph.D. Dissertation, University of Minnesota, 1993.

[62] Y. Miyano, A. Kimura, M. Kuroda, A. Matsushita, A. Yamasaki, Y. Yamaguchi, A. Yoshizawa, Y. Tateishi, "Henry's law constants and infinite dilution activity coefficients of propane, propene, butane, 2-methylpropane, 1-butene, 2-methylpropene, trans-2-butene, cis-2-butene, 1,3-butadiene, dimethyl ether, chloroethane, and 1,1difluoroethane in benzene, toluene, $o$-xylene, $m$-xylene, $p$-xylene, and styrene," J. Chem. Eng. Data, vol. 52, pp. 291-297, 2007.

[63] L. R. Field, E. Wilhelm and R. Battino, "Solubility of gases in liquids. 6. Solubility of nitrogen, oxygen, carbon monoxide, carbon dioxide, methane, and carbon tetrafluoride in methylcyclohexane and toluene at 283 to $313 \mathrm{~K}$," J. Chem. Thermodyn., vol. 6, pp. 237-243, 1974.

[64] M. H. Abraham, J. Gil-Lostes, W. E. Acree, Jr., J. E. ComettoMuniz and W. S. Cain, "Solvation parameters for mercury and mercury(II) compounds: calculation of properties of environmental interest," J. Environ. Monitor., vol. 10, pp. 435-442, 2008.

[65] A. M. Zissimos, M. H. Abraham, M. C. Barker, K. J. Box and K. Y. Tam, "Calculation of Abraham descriptors from solventwater partition coefficients in four different systems; evaluation of different methods of calculation," J. Chem. Soc., Perkin Trans. 2, no. 3, pp. 470-477, 2002.
[66] J. A. Waters, G. A. Mortimer and H. E. Clements, "Solubility of some light hydrocarbons and hydrogen in some organic solvents," J. Chem. Eng. Data, vol. 15, pp. 174-176, 1970.

[67] J. Li, T. Zhu, G. D. Hawkins, P. Winget, D. A. Liotard, C. J. Cramer and D. G. Truhlar, "Extension of the platform of applicability of the SM5.42R universal solvation model," Theor. Chem. Accounts, vol. 103, pp. 9-63, 1999.

[68] E. R. Thomas, B. A. Newman, T. C. Long, D. A. Wood and C. A. Eckert, "Limiting activity coefficients of nonpolar and polar solutes in both volatile and nonvolatile solvents by gas chromatography," J. Chem. Eng. Data, vol. 27, pp. 399-405, 1982.

[69] I. Landau, A. J. Belfer and D. C. Locke, "Measurement of limiting activity coefficients using non-steady-state gas chromatography," Ind. Eng. Chem. Res., vol. 30, pp. 1900-1906, 1991.

[70] H. C. Van Ness and B. D. Smith, "Excess Gibbs energy. 1Chlorobutane - toluene system," Int. DATA Ser., Sel. Data Mix., Ser. A, p. 82, 1983.

[71] S. Endo and T. C. Schmidt, "Partitioning properties of linear and branched ethers: Determination of linear solvation energy relationship (LSER) descriptors," Fluid Phase Equilibr., vol. 246, pp. 143$152,2006$.

[72] S. L. Oswal and D. D. Deshpande, "Excess Gibbs energy. Oxolanetoluene system," Int. DATA Ser., Sel. Data Mix., Ser. A, p. 18, 1988.

[73] J. H. Park, A. Hussam, P. Couasnon, D. Fritz and P. W. Carr, "Experimental reexamination of selected partition coefficients from Rohrschneider's data set," Anal. Chem., vol. 59, pp. 1970-1976, 1987.

[74] M. H. Abraham, J. Gil-Lostes, J. E. Cometto-Muniz, W. S. Cain, C. F. Poole, S. N. Atapattu, R. J. Abraham and P. Leonard, "The hydrogen bond acidity and other descriptors for oximes, New J. Chem., vol. 33, pp. 76-81, 2009.

[75] H. Renon, "Excess Gibbs energy. Nitroethane-toluene system," Int. DATA Ser., Sel. Data Mix., Ser. A, p. 3. 1974.

[76] M. H. Abraham, J. M. R. Gola, J. E. Cometto-Muniz and W. S. Cain, "The solvation properties of nitric oxide," J. Chem. Soc., Perkin Trans. 2, no. 10, pp. 2067-2070, 2000.

[77] M. H. Abraham and A. Nasehzadeh, "Thermodynamics of solution of gaseous tetramethyltin in 36 solvents. Comparison of experimental results with cavity-theory calculations," J. Chem. Soc., Faraday Trans. 1, vol. 77, pp. 321-339, 1981.

[78] S. M. Ashraf and D. H. L. Prasad, "Isothermal phase equilibrium studies on the binary mixtures of some alkylbenzenes," Phys. Chem. Liq., vol. 37, pp. 373-380, 1999.

[79] P. Vrbka, D. Rozbroj and V. Dohnal, "Limiting activity coefficients in binary mixtures of 1-alkanols and toluene," Fluid Phase Equilibr., vol. 209, pp. 265-280, 2003.

[80] P. Oracz, "Excess Gibbs energy. 2-Methyl-2-propanol - toluene system,” Int. DATA Ser., Sel. Data Mix., Ser. A, p. 246, 1989.

[81] K. D. Kassmann and H. Knapp, "Vapor-liquid equilibria for binary and ternary mixtures of benzene, toluene and $n$-butyraldehyde," Ber.Bunsen-Ges., vol. 90, pp. 452-458, 1986.

[82] C. Alonso-Tristan, J. J. Segovia, C. R. Chamorro and M. A. Villamanan, "Experimental investigation of the vapour-liquid equilibrium of binary and ternary mixtures containing dibutyl ether (DBE), cyclohexane and toluene at 313.15 K," Fluid Phase Equilibr., vol. 245, pp. 57-62, 2006.

[83] Y. Marcus, "Solubilities of buckminsterfullerene and sulfur hexafluoride in various solvents," J. Phys. Chem. B, vol. 101, pp. 8617-8623, 1997.

[84] R. G. Makitra, V. B. Moin and T. I. Politanskaya, "Solubility of trifluorochloromethane, difluorodichloromethane, trifluoroethylene, and trifluorochloroethylene in toluene and dichlorofluoromethane (Freon 21) in N-methylpyrrolidinone," Zh. Priklad. Khim., vol. 57, pp. 1648-1651, 1984.

[85] S. Horstmann, H. Gardeler, R. Boelts and J. Gmehling, “ Vaporliquid equilibria and excess enthalpy data for the binary systems benzene + benzonitrile and toluene + benzonitrile covering the temperature range from 323.15 to $413.15 \mathrm{~K}$," J. Chem. Eng. Data, vol. 44, pp. 539-543, 1999.

[86] W. E. Acree, Jr. and M. H. Abraham, "Solubility predictions for crystalline polycyclic aromatic hydrocarbons (PAHs) dissolved in organic solvents based upon the Abraham general solvation model," Fluid Phase Equilibr., vol. 201, pp. 245-258, 2002. 
[87] C. Z. Jiang, F. J. Hsu and V. Fried, "Solubility of iodine in electron-donating solvents at various temperatures," J. Chem. Eng. Data, vol. 28, pp. 75-78, 1983.

[88] K. R. Hoover, W. E. Acree, Jr. and M. H. Abraham, "Correlation of the solubility behavior of crystalline 1-Nitronaphthalene in organic solvents with the Abraham solvation parameter model," J. Solution Chem., vol. 34, pp. 1121-1133, 2005.

[89] C. I. Monarrez, D. M. Stovall, J. H. Woo, P. Taylor and W. E. Acree, Jr., "Solubility of 9-fluorenone in organic nonelectrolyte solvents: comparison of observed versus predicted values based upon mobile order theory," Phys. Chem. Liq., vol. 41, pp. 73-80, 2003.

[90] W. E. Acree, Jr. and M. H. Abraham, "Solubility of crystalline nonelectrolyte solutes in organic solvents: mathematical correlation of benzil solubilities with the abraham general solvation model," $J$. Solution Chem., vol. 31, pp. 293-303, 2002.

[91] C. E. Green, M. H. Abraham, W. E. Acree, Jr., K. M. De Fina and T. L. Sharp, "Solvation descriptors for pesticides from the solubility of solids: diuron as an example," Pest Manage. Sci., vol. 56, pp. 1043-1053, 2000.

[92] K. A. Fletcher, K. S. Coym, L. E. Roy, C. E. Hernandez, M. E. R. McHale and W. E. Acree, Jr., "Solubility of thioxanthen-9-one in organic nonelectrolyte solvents: comparison of observed versus predicted values based upon mobile order theory (MOT)," Phys. Chem. Liq., vol. 35, pp. 243-252, 1998.

[93] M. H. Abraham, L. Honcharova, S. A. Rocco, W. E. Acree, Jr, K. M. De Fina, "The lipophilicity and hydrogen bond strength of pyridine-N-oxides and protonated pyridine-N-oxides," $N$ J. Chem., vol. 35, pp. 930-936, 2011.

[94] K. B. Flanagan, K. R. Hoover, O. Garza, A. Hizon, T. Soto, N. Villegas, W. E. Acree, Jr. and M. H. Abraham, "Mathematical correlation of 1-chloroanthraquinone solubilities in organic solvents with the Abraham solvation parameter model," Phys. Chem. Liq., vol. 44, pp. 377-386, 2006.

[95] D. M. Stovall, W. E. Acree, Jr. and M. H. Abraham, "Solubility of 9-fluorenone, thianthrene and xanthene in organic solvents," Fluid Phase Equilibr., vol. 232, 1-2, pp. 113-121, 2005.

[96] K. R. Hoover, K. Pop, W. E. Acree, Jr. and M. H. Abraham, "Solubility of crystalline nonelectrolyte solutes in organic solvents: mathematical correlation of 3-chlorobenzoic acid solubilities with the Abraham solvation parameter model," South Afr. J. Chem., vol. 58, pp. 25-29, 2005.

[97] K. R. Hoover, D. M. Stovall, E. Pustejovsky, R. Coaxum, K. Pop, W. E. Acree, Jr. and M. H. Abraham, "Solubility of crystalline nonelectrolyte solutes in organic solvents - mathematical correlation of 2-methoxybenzoic acid and 4-methoxybenzoic acid solubilities with the Abraham solvation parameter model," Can. J. Chem., vol. 82, pp. 1353-1360, 2004.

[98] A. K. Charlton, C. R. Daniels, R. M. Wold, E. Pustejovsky, W. E. Acree, Jr., M. H. Abraham, "Solubility of crystalline nonelectrolyte solutes in organic solvents: mathematical correlation of 3nitrobenzoic acid solubilities with the Abraham general solvation model," J. Mol. Liq., vol. 116, pp. 19-28, 2004.

[99] K. R. Hoover, R. Coaxum, E. Pustejovsky, D. M. Stovall, W. E. Acree, Jr. and M. H Abraham, "Thermochemical behavior of dissolved carboxylic acid solutes: part 4 - mathematical correlation of 4-nitrobenzoic acid solubilities with the Abraham solvation parameter model," Phys. Chem. Liq., vol. 42, pp. 339-347, 2004.

[100] R. Coaxum, K. R. Hoover, E. Pustejovsky, D. M. Stovall, W. E. Acree, Jr. and M. H. Abraham, "Thermochemical behavior of dissolved carboxylic acid solutes: Part 3 - Mathematical correlation of 2-methylbenzoic acid solubilities with the Abraham solvation parameter model," Phys. Chem. Liq., vol. 42, pp. 313-322, 2004.

[101] M. H. Abraham, "Hydrogen bond and other descriptors for thalidomide and its $\mathrm{N}$-alkyl analogs; prediction of physicochemical and biological properties," Eur. J. Pharm. Sci., vol. 21, pp. 465469, 2004

[102] C. R. Daniels, A. K. Charlton, R. M. Wold, W. E. Acree, Jr. and M. H. Abraham, "Thermochemical behavior of dissolved carboxylic acid solutes: Solubilities of 3-methylbenzoic acid and 4chlorobenzoic acid in organic solvents," Can. J. Chem., vol. 81, pp. 1492-1501, 2003.

[103] M. H. Abraham, M. Amin and A. M. Zissimos, "The lipophilicity of Sudan I and its tautomeric forms," Phys. Chem. Chem. Phys., vol. 4, pp. 5748-5752, 2002.
[104] K. M. De Fina, T. L. Sharp, I. Chuca, M. A. Spurgin, W. E. Acree, Jr., C. E. Green and M. H. Abraham, "Solubility of the pesticide monuron in organic nonelectrolyte solvents. Comparison of observed versus predicted values based upon Mobile Order theory," Phys. Chem. Liq., vol. 40, pp. 255-268, 2002.

[105] K. M. De Fina, T. L. Sharp, M. A. Spurgin, I. Chuca, W. E. Acree, Jr., C. E. Green and M. H. Abraham, "Solubility of the pesticide diuron in organic nonelectrolyte solvents. Comparison of observed vs. predicted values based upon mobile order theory ," Can. J. Chem., vol. 78, pp. 184-190, 2000.

[106] E. L. Heric and C. D. Posey, "Interaction in nonelectrolyte solutions. Solubility of naphthalene in some mixed solvents containing benzene," J. Chem. Eng. Data, vol. 9, pp. 35-43, 1964.

[107] W. Chang, "Solubilities of biphenyl, naphthalene, perfluorobiphenyl, perfluoronaphthalene, and hexachloroethane in nonelectrolytes," Ph.D. Dissertation, North Dakota State University, Fargo, North Dakota, 1969.

[108] D. M. Stovall, C. Givens, S. Keown, K. R. Hoover, E. Rodriguez, W. E.Acree, Jr. and M. H. Abraham, "Solubility of crystalline nonelectrolyte solutes in organic solvents: Mathematical correlation of ibuprofen solubilities with the Abraham solvation parameter model," Phys. Chem. Liq., vol. 43, pp. 261-268, 2005.

[109] L. E. Roy, C. E. Hernandez, K. M. De Fina and W. E. Acree, Jr. "Thermodynamics of mobile order theory. Part 4: comparison of experimental and predicted solubilities for trans-stilbene," Phys. Chem. Liq., vol. 38, pp. 333-343, 2000.

[110] W. E. Acree, Jr. and M. H. Abraham, "Solubility predictions for crystalline nonelectrolyte solutes dissolved in organic solvents based upon the Abraham general solvation model," Can. J. Chem., vol. 79, pp. 1466-1476, 2001.

[111] K. M. De Fina, C. Ezell and W. E. Acree, Jr., "Solubility of ferrocene in organic nonelectrolyte solvents. Comparison of observed versus predicted values based upon mobile order theory," Phys. Chem. Liq., vol. 39, pp. 699-710, 2001.

[112] J. M. Berryman and E. L. Heric, "Solubility of p-dibromobenzene at $25^{\circ}$ in some mixed solvents," J. Chem. Eng. Data, vol. 12, pp. 249-252, 1967.

[113] Z. Zou, L. Dang, P. Liu and H. Wei, "Solubility of fluorene in different solvents from $278.98 \mathrm{~K}$ to $338.35 \mathrm{~K}$," J. Chem. Eng. Data, vol. 52, pp. 1501-1502, 2007.

[114] Ya. I. Korenman, "Distribution of chlorophenols between organic solvents and water," Zh. Priklad. Khim., vol. 47, pp. 2079-2083, 1974.

[115] Ya. I. Korenman and T. V. Makarova, "Extraction of 2-halophenols from aqueous solutions," Zh. Priklad. Khim., vol. 47, pp. 1624$1628,1974$.

[116] Ya. I. Korenman, "Solubility parameters and extraction of phenols," Russ. J. Phys. Chem., vol. 46, pp. 334-336, 1972.

[117] Ya. I. Korenman, E. B. Kotelyanskaya and T. A. Nefedova, "Extraction of mononitrophenols from aqueous solutions," Zh. Priklad. Khim., vol. 49, pp. 1112-1114, 1976.

[118] Ya. I. Korenman, T. A. Nefedova and R. I. Dyukova, "Extraction of phenol, resorcinol, and their 2,4-dinitrosubstituted derivatives," Zh. Priklad. Khim., vol. 50, pp. 2736-2738, 1977.

[119] D. S. Abrams and J. M. Prausnitz, "Distribution of phenolic solutes between water and nonpolar organic solvents," J. Chem. Thermodyn., vol. 7, pp. 61-72, 1975

[120] Ya. V. Arro and L. N. Melder, "Calculation of partition coefficients for polar substances between a mixture of organic solvents and water," Russ. J. Phys. Chem., vol. 50, pp. 1553-1555, 1976.

[121] Ya. I. Korenman, "Extraction of xylenols," Zh. Priklad. Khim., vo. 46, pp. 380-384, 1973

[122] Ya. I. Korenman, "Correlation between distribution constants of related substances," Russ. J. Phys. Chem., vol. 46, pp. 1286-1288, 1972.

[123] J. Arro and L. Molder, "Activity coefficients of dihydric phenols in water from their distribution between two liquid phases," Russ. J. Phys. Chem., vol. 49, pp. 635-636, 1975.

[124] I. M. Korenman and T. M. Kochetkova, "Partition of toluidines between some organic solvents and water," Trudy po Khimii $i$ Khim. Tekhnol., pp. 55-58, 1969.

[125] Ya. I. Korenman and G. M. Smol'skii, "Extraction removal of nitroanilines from aqueous solutions," Zh. Priklad. Khim., vol. 64, pp. 1044-1048, 1991. 
[126] I. M. Korenman and L. N. Karyakina, "Effect of temperature on the extracton of naphthylamines," Russ. J. Phys. Chem., vol. 47, no. 6, pp. 850-852, 1973.

[127] W. Robak, W. Apostoluk and P. Maciejewski, "Analysis of liquidliquid distribution constants of nonionizable crown ethers and their derivatives," Anal. Chim. Acta, vol. 569, pp. 119-131, 2006.

[128] Y. Takeda, K. Endō, D. Yoshiyama, K. Watanabe, N. Fukada and S. Katsuta, "Quantitative elucidation of macrocyclic effect on extraction-ability and -selectivity. benzo-18-crown-6 and its openring analog 1,2-bis[2-(2-methoxyethoxy)ethoxy]-benzene-alkali metal picrate systems," J. Mol. Liq., vol. 130, pp. 21-28, 2006.

[129] Ya. I. Korenman, I. I. Butyaeva and M. M. Gel'fand, "Extraction of naphthols from aqueous solutions," Zh. Priklad. Khim., vol. 47, no. 2, pp. 473-475, 1974.

[130] I. M. Korenman and Z. G. Chernorukova, "Distribution of normal alcohols between organic solvents and water ," Zh. Priklad. Khim., vol. 47, no. 11, pp. 2523-2526, 1974.

[131] I. Tohyama and K. Otozai, "Solvent extraction of halogens," Fresenius' Zeitschrift Anal. Chem., vol. 293, pp. 282-285, 1978.

[132] I. M. Korenman and T. D. Klyukvina, "Extraction of methylfurfural from aqueous solutions," Zh. Priklad. Khim., vol. 45, pp. 2572-2574, 1972.

[133] Ya. I. Korenman and A. A. Gorokhov, "Certain characteristics of the extraction of di(hydroxyphenyl)propane," Russ. J. Phys. Chem., vol. 47, pp. 1157-1158, 1973.

[134] I. M. Korenman, N. Yu. Gurevich and T. G. Kulagina, "Extraction of some n-alkylamines from aqueous solutions," Zh. Priklad. Khim., vol. 46, pp. 683-684, 1973.
[135] Ya. I. Korenman, "Effect of substituents of different nature on distribution coefficients for phenols in water-organic solvents systems," Zhur. Fiz. Khim., vol. 56, pp. 748-751, 1982.

[136] I. M. Korenman and M. I. Gryaznova, "Distribution of $\beta$-diketones between organic solvents and water," Zhur. Anal. Khim. vol. 29, pp. 964-969, 1974.

[137] J. G. Mason and I. Lipschitz, "Thermodynamics of the partition of 8-quinolinol between several organic solvents and aqueous buffers," Talanta, vol. 18, pp. 1111-1115, 1971.

[138] P. C. Ray and P. K. Das, "Measurement of partition coefficients of substituted benzoic acids between two immiscible solvents by hyper-Rayleigh scattering," J. Phys. Chem., vol. 100, pp. 15631$15633,1996$.

[139] Ya. I. Korenman and T. P. Koroleva, "Extraction of hydroxybenzoic acids and some of their derivatives from aqueous solutions," Zh. Priklad. Khim., vol. 48, pp. 1413-1415, 1975.

[140] M. Góral, B. Wisniewska-Goclowska and A. Maczynski, "Recommended liquid-liquid equilibrium data. Part 3. Alkylbenzene-water systems," J. Phys. Chem. Ref. Data, vol. 33, pp. 1159-1188, 2004.

[141] C. Mintz, M. Clark, W. E. Acree, Jr. and M. H. Abraham, "Enthalpy of solvation correlations for gaseous solutes dissolved in water and in 1-octanol based on the Abraham model," J. Chem. Inf. Model., vol. 47, pp. 115-121, 2007.

[142] C. Mintz, M. Clark, K. Burton, W. E. Acree, Jr. and M. H. Abraham, "Enthalpy of solvation correlations for gaseous solutes dissolved in toluene and carbon tetrachloride based on the Abraham model," J. Solution Chem., vol. 36, pp. 947-966, 2007.

(C) Stephens et al.; Licensee Bentham Open.

This is an open access article licensed under the terms of the Creative Commons Attribution Non-Commercial License (http://creativecommons.org/licenses/ by-nc/3.0/) which permits unrestricted, non-commercial use, distribution and reproduction in any medium, provided the work is properly cited. 